\title{
Higgs Searches Beyond the Standard Model
}

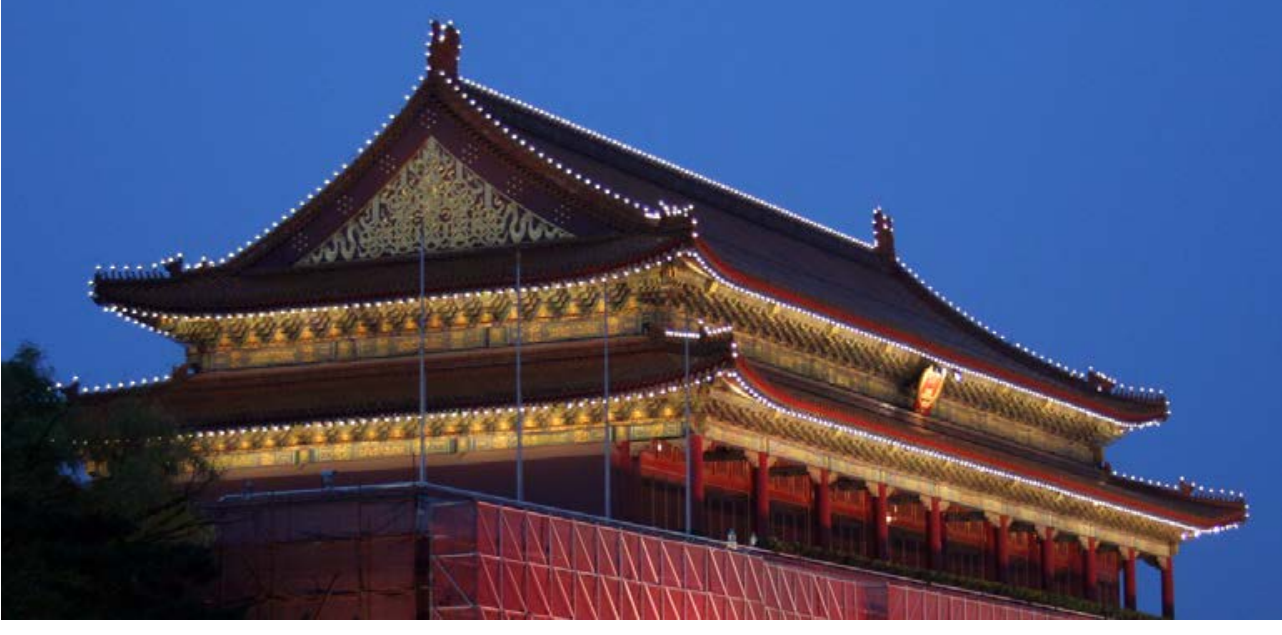

Rainer Mankel

Deutsches Elektronen-Synchrotron (DESY) 汉堡 on behalf of the ATLAS, CDF, CMS and DO Collaborations

PIC2013 Conference,
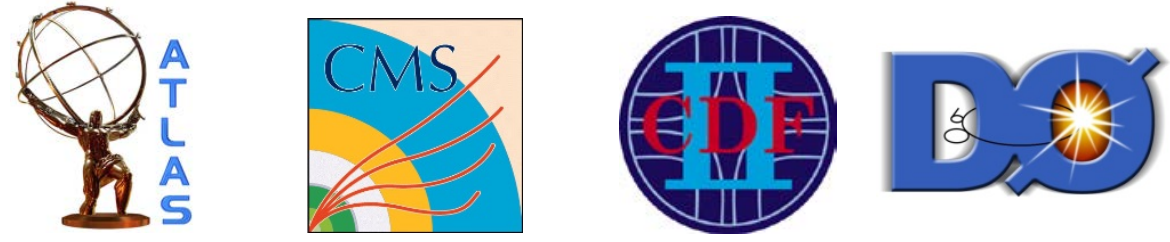

IHEP Beijing,

3-7 Sept 2013 
- Most relevant questions after discovery of a Higgs boson at $\sim 125 \mathrm{GeV}$ :

- $\quad$ properties of this Higgs boson, couplings etc $\rightarrow$ see talks by James, Romain \& Elisabetta

- structure of the Higgs sector

- At the level of current measurements, the observed state is compatible with the Standard Model Higgs

- but SM features quadratically divergent self-energy corrections at high energies (Hierarchy problem)

- many other open questions: dark matter, naturalness (" $\mu$ problem"), CP violation in early universe

- Even with SM-like tree-level production mechanisms, there is still plenty of room for non-SM decays of the $\mathrm{H}(125)$

- $\mathrm{BR}_{\mathrm{BSM}}<52 \%$ at $95 \% \mathrm{CL}$

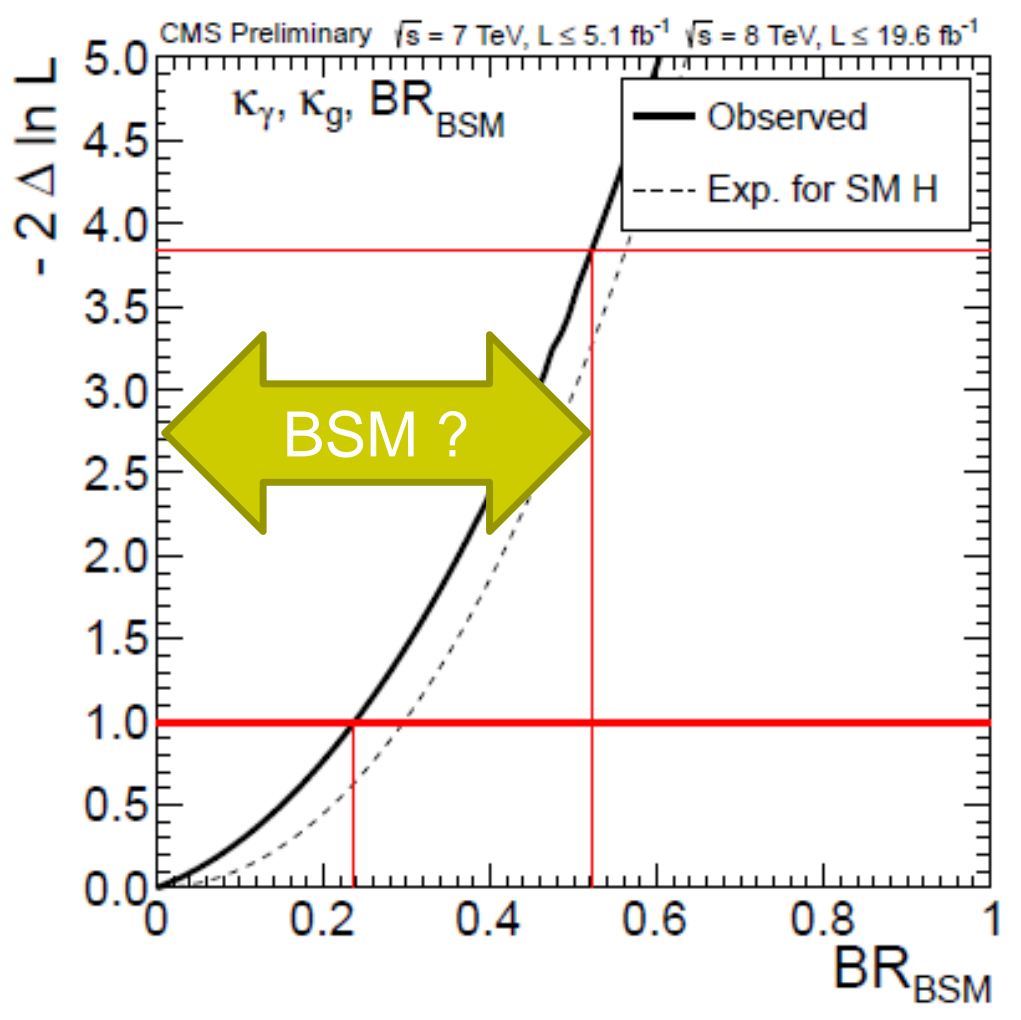

Searching additional Higgs states is potentially the fastest way of answering these questions 


\section{Extended Higgs Sectors}

\section{MSSM}

- two complex scalar doublet fields

- five physical Higgs bosons

\section{HDM Models}

- more general formulation of model with two scalar fields

- MSSM is a type-II 2HDM

- $\mathrm{CP}$ violation and FCNC possible

Standard Model
- $\quad$ single complex scalar doublet
field
- $\quad$ one physical Higgs state $(\mathrm{H})$

\section{NMSSM}

- two complex scalar doublet fields + additional singlet

- $\quad$ seven physical Higgs states
Additional SM-like Higgs

- $\quad$ high mass searches

\section{Fermiophobic}

- not coupling to fermions

\section{Hidden Sector}

- invisible Higgs

- dark SUSY

\section{Many others}




\section{The Experiments}
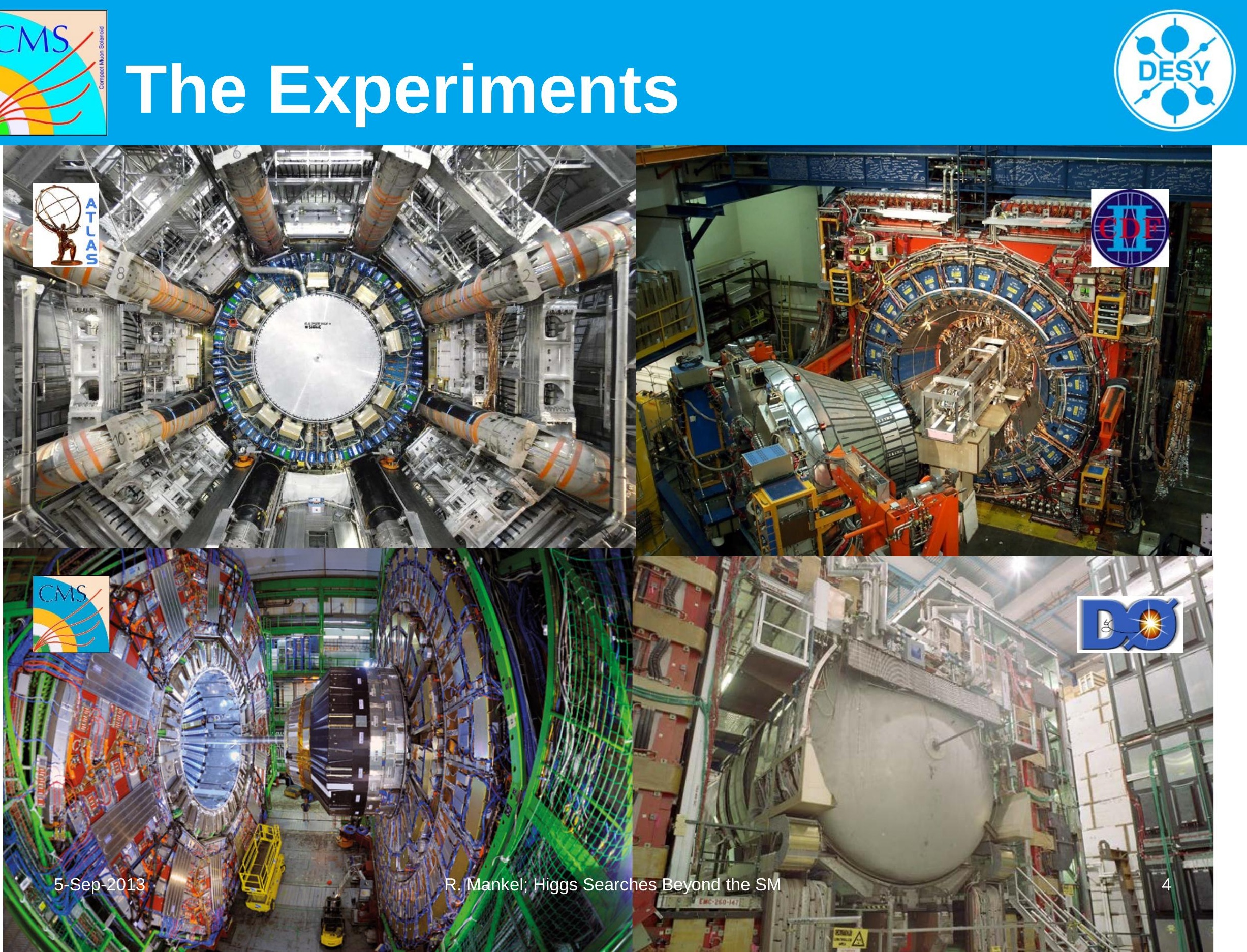
MSSM Higgs Bosons 
- MSSM features two complex Higgs doublets

$\rightarrow$ Five physical Higgs bosons

- three neutral: $\mathrm{h}, \mathrm{H}, \quad \mathrm{A}$

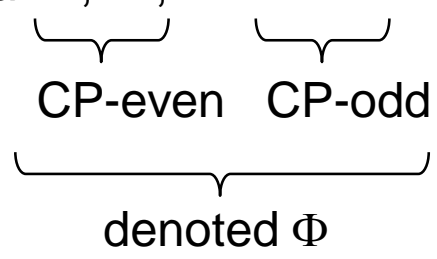

- two charged: $\mathrm{H}^{ \pm}$

- At tree level, MSSM Higgs sector is governed by

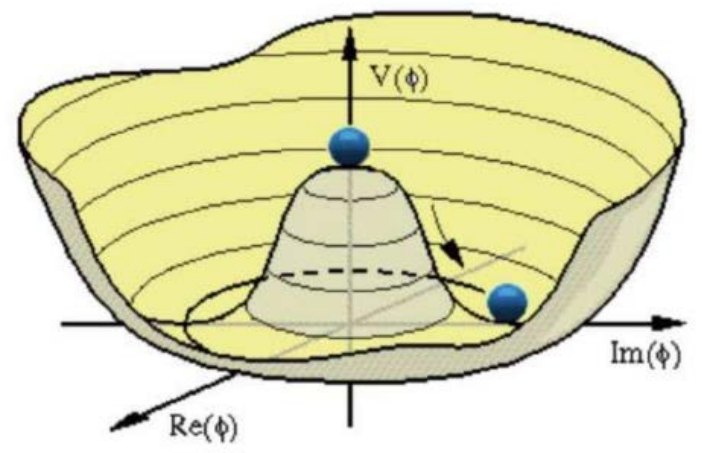
two parameters:

- $\mathrm{m}_{\mathrm{A}}$

- $\tan \beta$ (ratio of vacuum expectation values of the two Higgs doublets)

- Beyond tree level, additional parameters enter via radiative corrections

$\rightarrow$ benchmark scenarios to compare different measurements (by default " $m_{h}{ }^{\max }$ ") 


\section{Higgs Masses in the MSSM}

- The mass of the CP-odd Higgs boson $A$ is usually degenerate with one of the CP-even bosons

- $\mathrm{m}_{\mathrm{A}} \approx \mathrm{m}_{\mathrm{H}}$ for $\mathrm{m}_{\mathrm{A}}>>\mathrm{m}_{\mathrm{h}}$ max

- $\mathrm{m}_{\mathrm{A}} \approx \mathrm{m}_{\mathrm{h}}$ for $\mathrm{m}_{\mathrm{A}} \ll \mathrm{m}_{\mathrm{h}}$ max

- With the exception of the $\mu \mu$ channel, this degeneracy cannot be resolved within the mass resolution

$\rightarrow \quad$ visible cross section effectively doubles

$\rightarrow$ Together with the effect of the Higgs coupling to b quarks, visible cross sections in b-associated production are typically enhanced by a factor of $\approx 2 \tan ^{2} \beta$

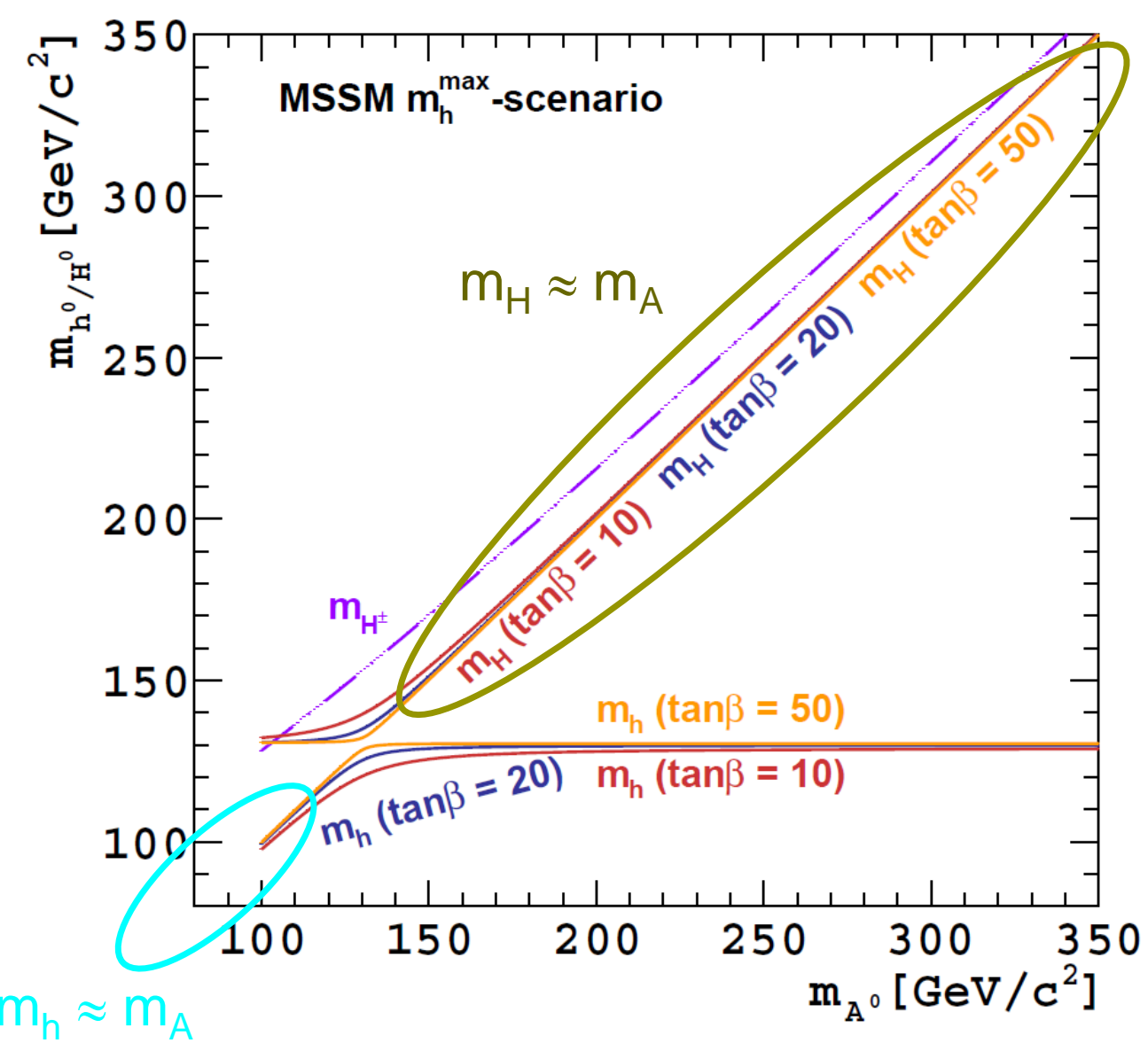




\section{MSSM Higgs Production \& Decay}

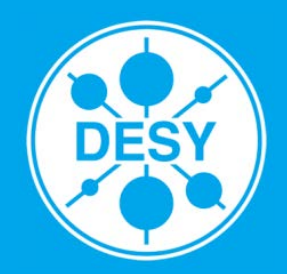

- Strong enhancement of cross section with increasing $\tan \beta$

- in particular due to associated production
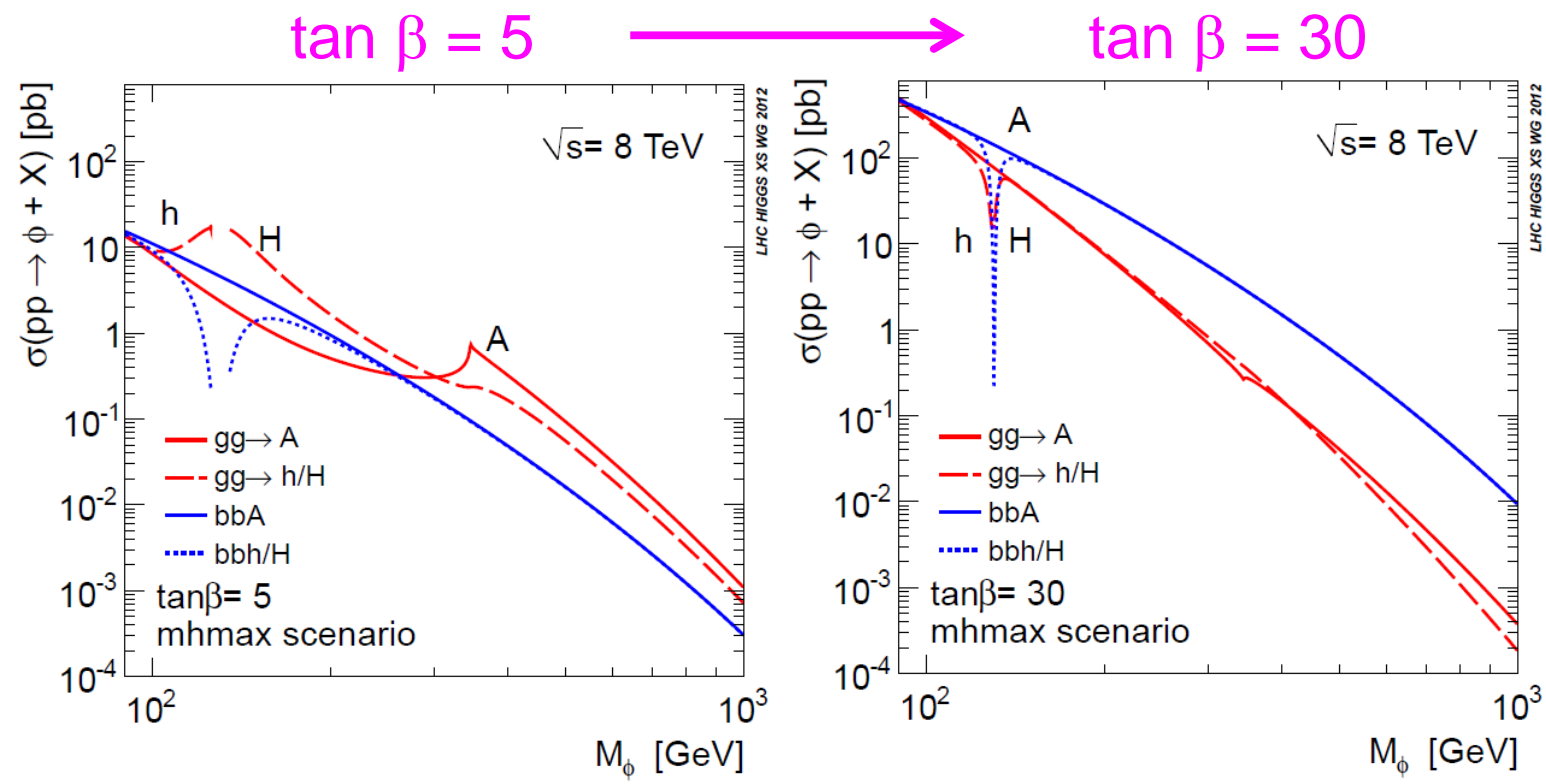

- Dominant decays of the neutral MSSM Higgs boson (at large $\tan \beta)$ :

- $b b(\sim 90 \%)$

- $\tau \tau(\sim 10 \%)$

$\rightarrow$ Unlike the SM, these decay modes may play important rôle even at high masses

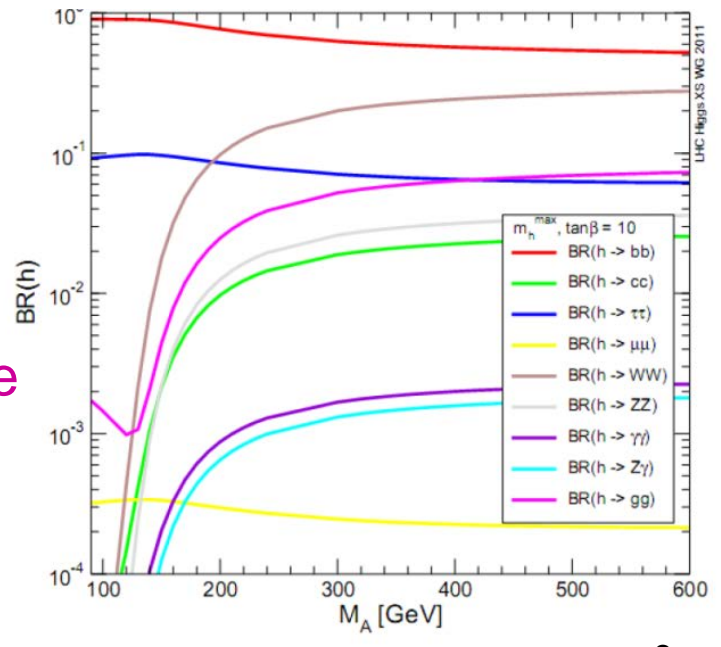


- Evaluate impact of $H(125)$ in $m_{h}{ }^{\bmod \pm}$ scenarios

- re-tuned version of $m_{h}{ }^{\text {max }}$ scenario, suits better the observed Higgs mass

- theoretical uncertainties taken into account
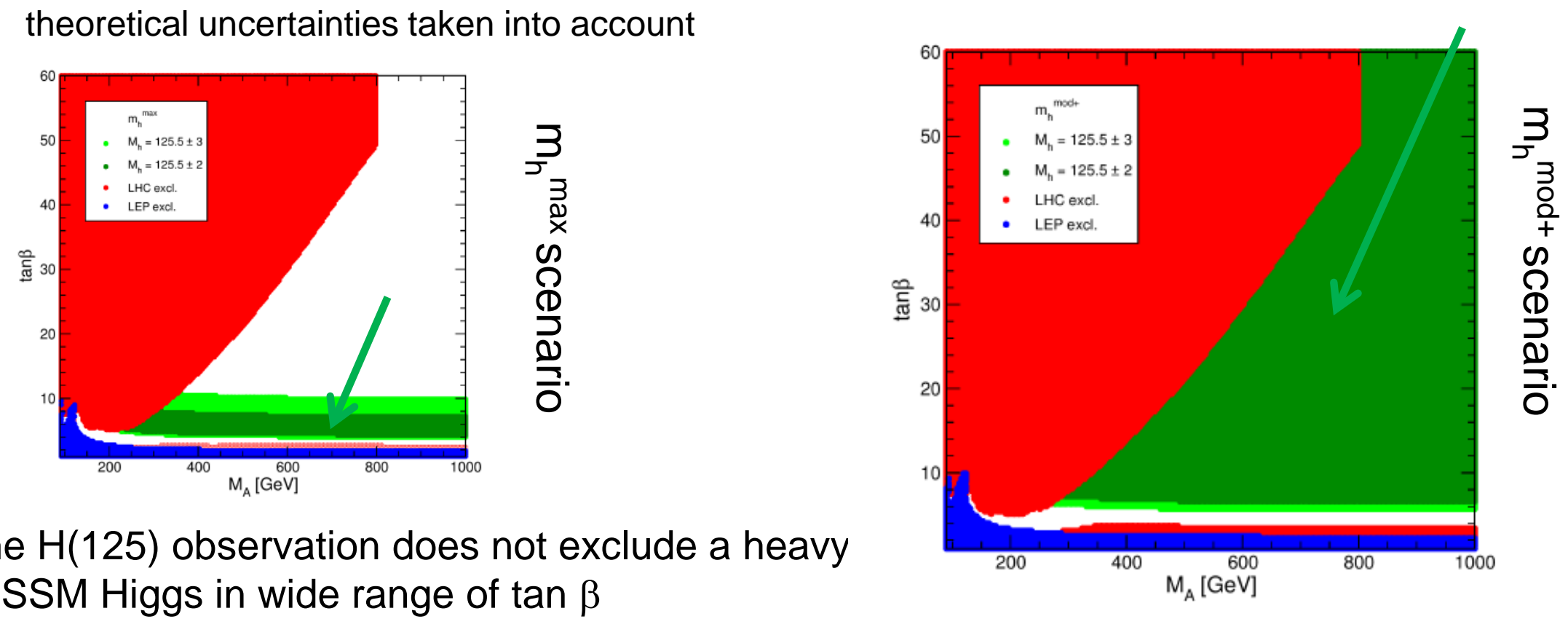

- The $H(125)$ observation does not exclude a heavy MSSM Higgs in wide range of $\tan \beta$

- At large $M_{A}\left(>>m_{Z}\right)$ the "light" MSSM Higgs boson ( $h$ ) becomes standard model-like (decoupling limit) $\rightarrow$ direct searches are essential

- Both SM and MSSM fit the current set of H(125) measurements equally well

P. Bechtle et al., arXiv:1211.1955 
- Good compromise between relatively large BR and manageable backgrounds

- To-date, analyses cover five of six possible $\tau \tau$ decay patterns

- $\quad \mathrm{e}+\mu, \mathrm{e}+\mathrm{had}, \mu+\mathrm{had}, \mathrm{had}+\mathrm{had}$ (ATLAS), $\mu+\mu$ (CMS)

- Mass of $\tau$ pair is reconstructed from visible $\tau$ decay products and missing $\mathrm{E}_{\mathrm{T}}$

- CMS: likelihood technique

- ATLAS: "Missing Mass Calculator" *

- Main backgrounds (in broad strokes - may differ from channel to channel):

- $\mathrm{Z} \rightarrow \tau \tau$ :

- embedding technique: take $Z \rightarrow \mu \mu$ from data, replace $\mu$ 's by simulated $\tau$ decays

No B-Tag

no b-tagged jet

- $\quad \mathrm{Z} \rightarrow \mathrm{ee} / \mu \mu$

- $t \bar{t}$ and di-boson

- QCD multijet, W+jets:

* A. Elagin et al., Nucl.Instrum.Meth. A654 (2011) 481-489 


\section{$\Phi \rightarrow \tau \tau:$ Mass Distributions (B-tag Category)}
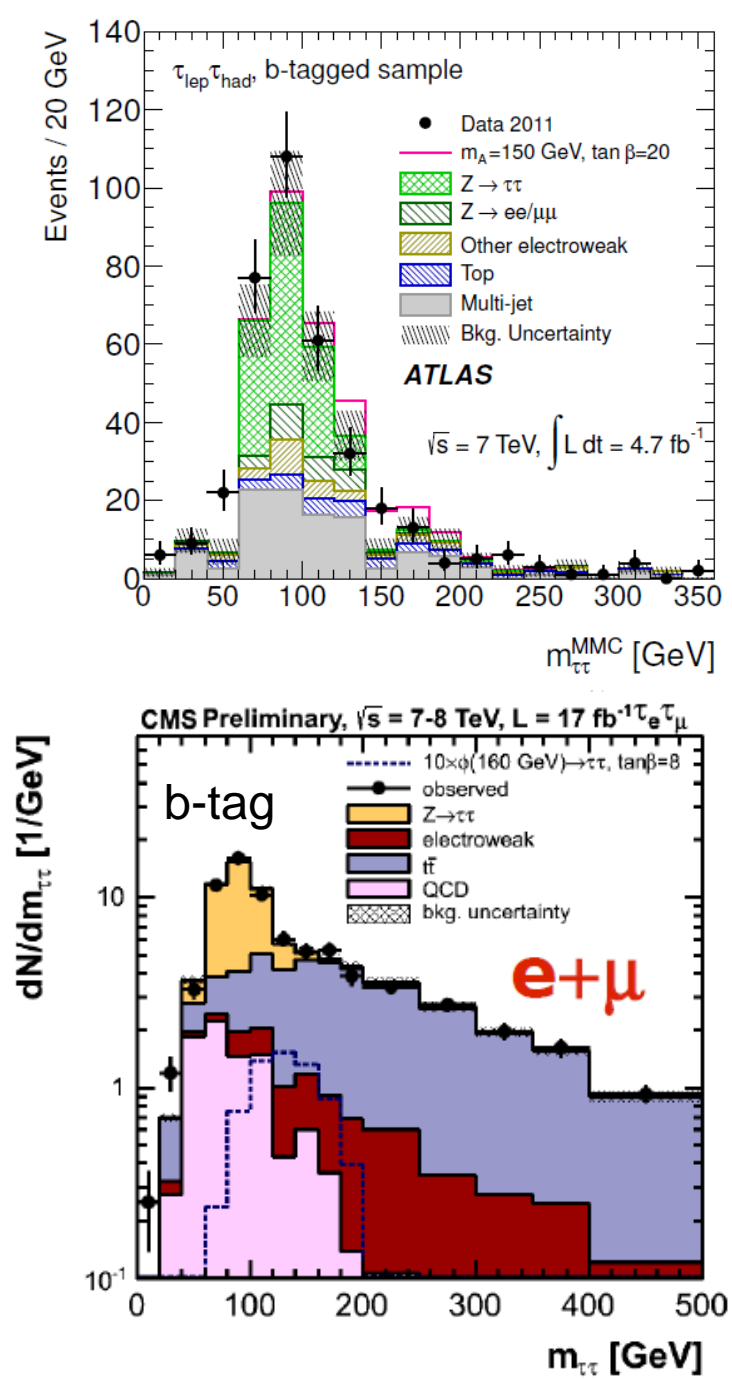
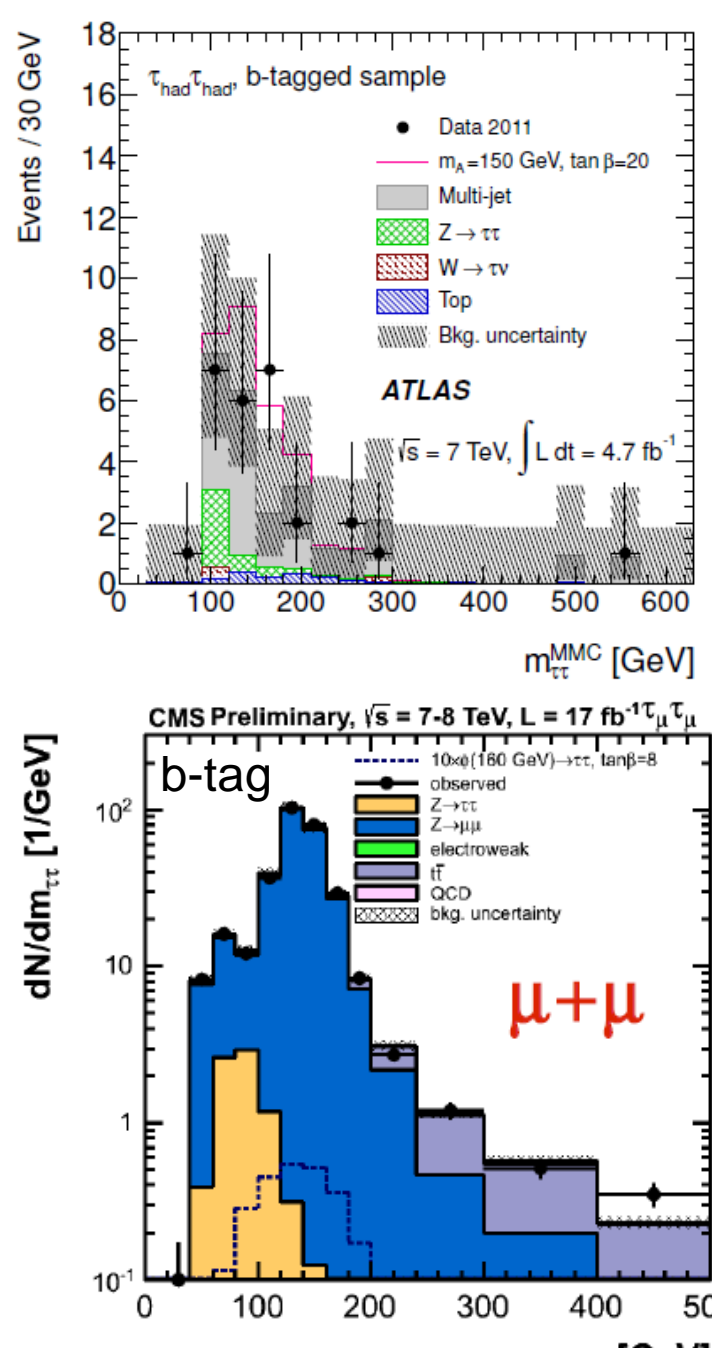

ATLAS JHEP 1302 (2013) 095

CMS PAS HIG-12-050

$\rightarrow$ Background compositions differ significantly across the various decay channels

$\rightarrow$ All distributions well described by background hypothesis 


\section{$\Phi \rightarrow \tau \tau:$ Results}

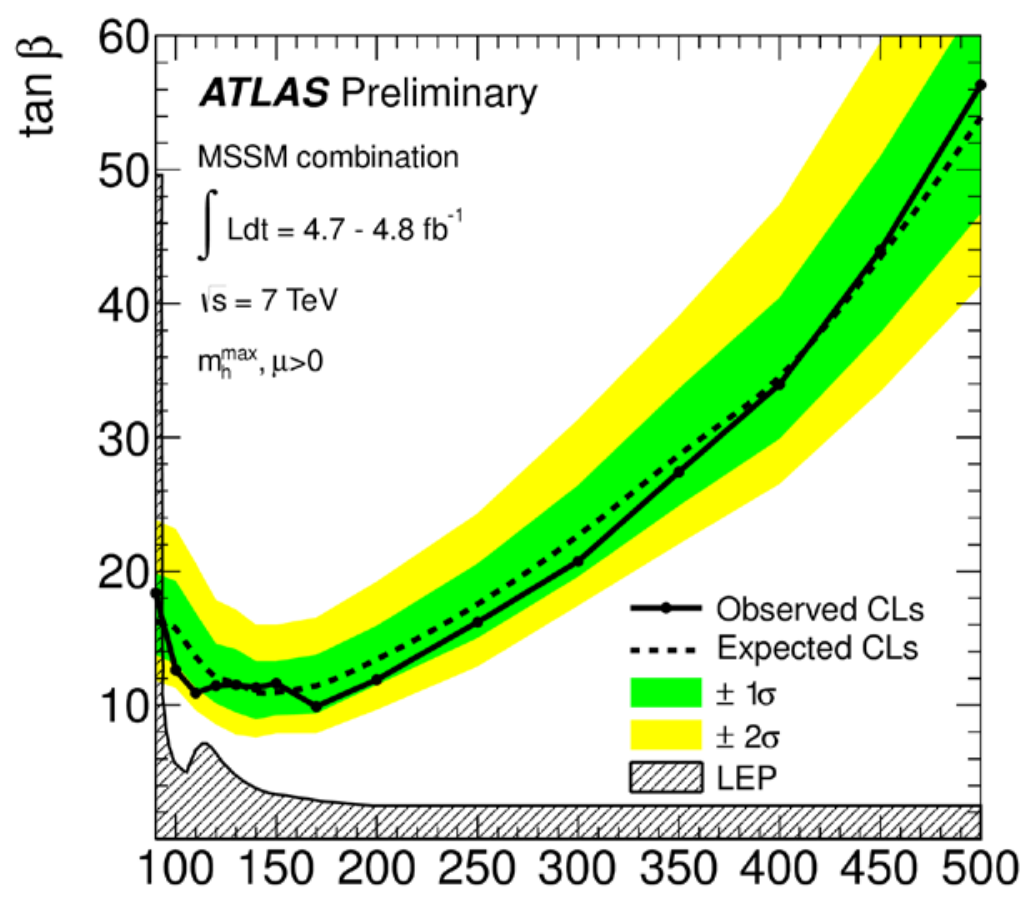

includes $\mu \mu$ channel (see next slide)

$$
\mathrm{m}_{\mathrm{A}}[\mathrm{GeV}]
$$

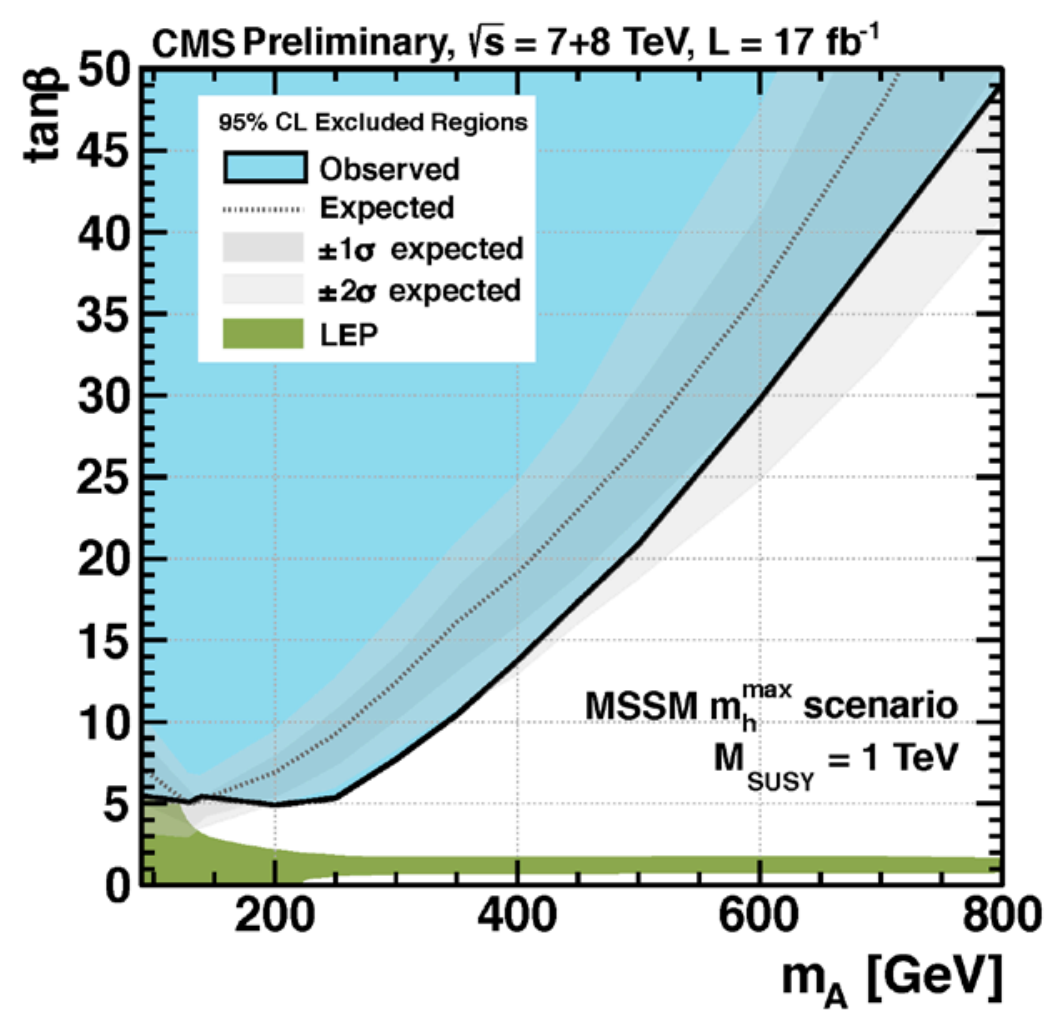

$\rightarrow$ Very low $\tan \beta$ upper limits $\left(\tan \beta<5\right.$ for $m_{A}<250 \mathrm{GeV}$ !)

- touching the LEP constraint at low $\mathrm{m}_{\mathrm{A}}$

$\rightarrow$ Addition of $8 \mathrm{TeV}$ data $\rightarrow$ extension of mass scale up to $800 \mathrm{GeV}$ (CMS) 
- Low BR, but also excellent mass resolution (close to $\Gamma_{\Phi}$ ) and manageable BG

- Dominant backgrounds: Drell-Yan, bbZ ${ }^{0}$, top

$\rightarrow$ Limits reach to $\sigma{ }^{*} \mathrm{BR}$ in the $20-100 \mathrm{fb}$ range

$\rightarrow$ significant constraints in $\left(m_{A}, \tan \beta\right)$ plane

\section{ATLAS JHEP 02 (2013) 095}

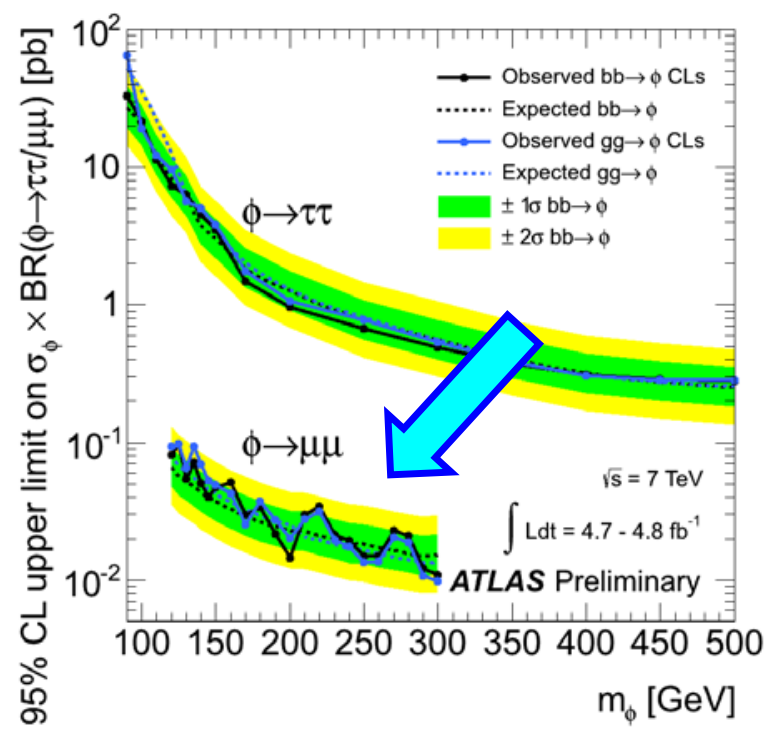

5-Sep-2013

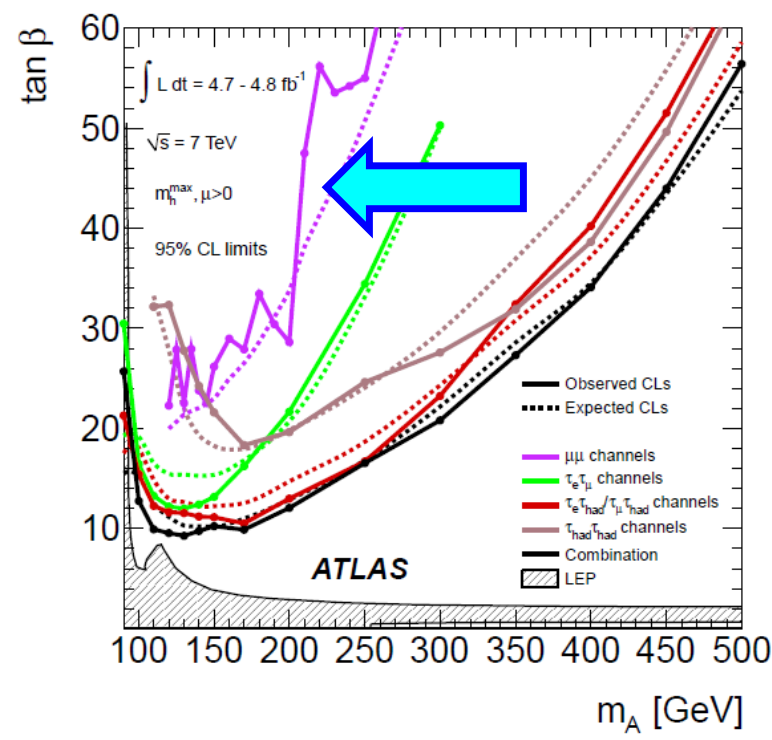

R. Mankel; Higgs Searches Beyond the SM

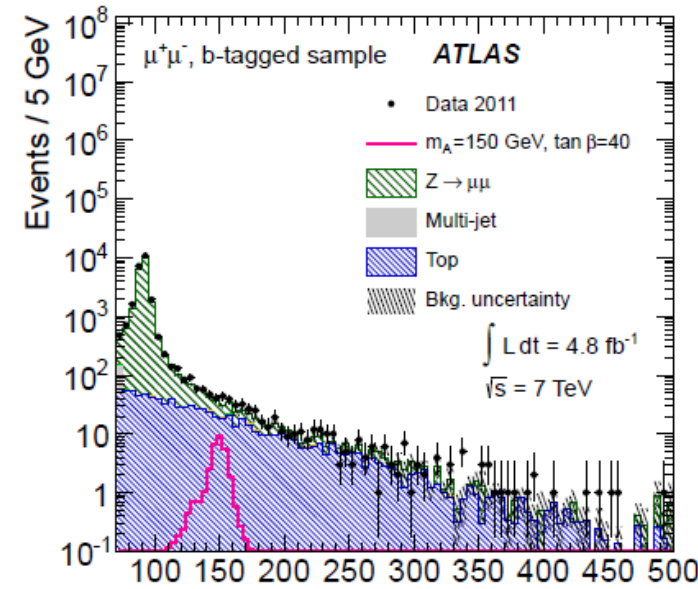

$\mathrm{m}_{\mu \mu}[\mathrm{GeV}]$

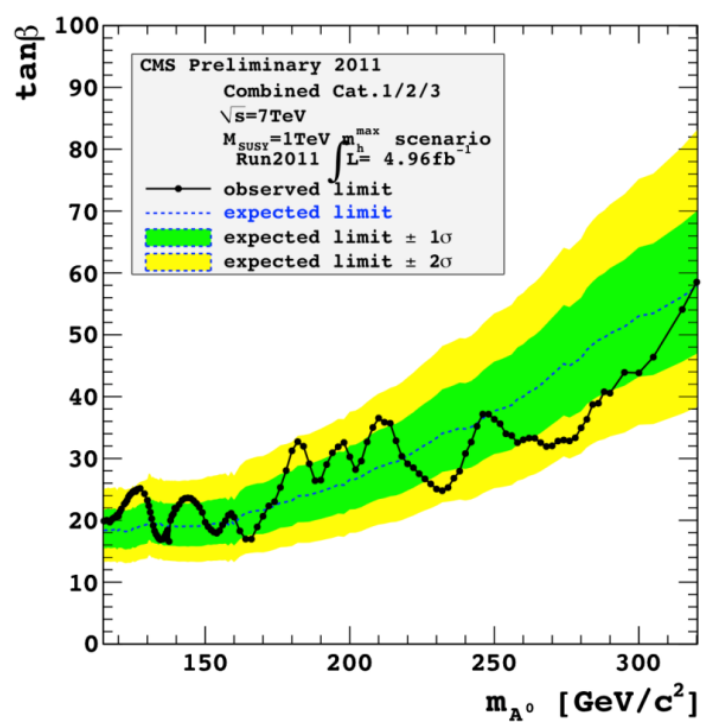

13 


\section{$\Phi \rightarrow b \bar{b}$ Search}

- Largest expected BR, but very difficult channel

- $\quad$ huge background from multi-jet QCD

- Search for associated production with at least one additional b quark

$\rightarrow$ enhancement if $\tan \beta>1$

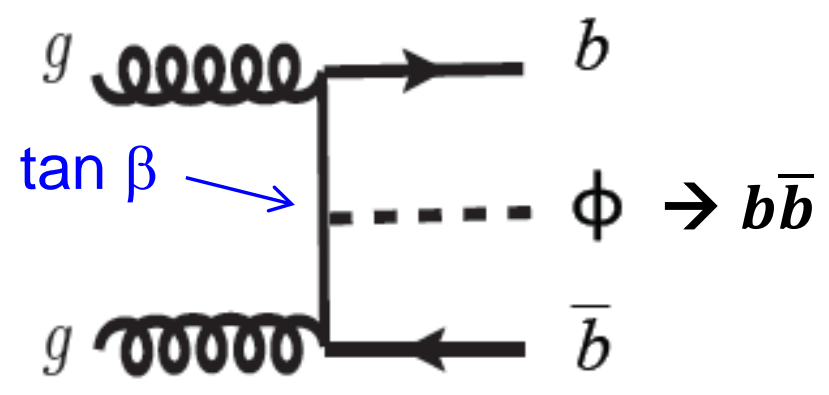

- Signature: $\geq$ three $b$-jets $+X$

in the final state

$\rightarrow \quad$ one of the most challenging triggers 


\section{$\Phi \rightarrow b \bar{b}:$ Tevatron Measurements}

\section{CDF+D0 Phys.Rev. D86 (2012) 091101}

- Search for MSSM Higgs boson decaying to b quarks, and produced with at least one additional b jet

- Background treatment:

- CDF: mass + global b-tag templates, derived from double btag sample with btag efficiency weights. Combination fitted to data.

- D0: fractional contributions of multi-jet processes determined by fitting $\mathrm{p}_{\mathrm{T}}$ distributions from simulation to the data.

$\rightarrow$ No signal seen over background expectation

- $\quad$ modest excesses of $\sim 2.8 \sigma$ (CDF) and $\sim 2.5 \sigma$ (D0)

Best Fit (with signal template)
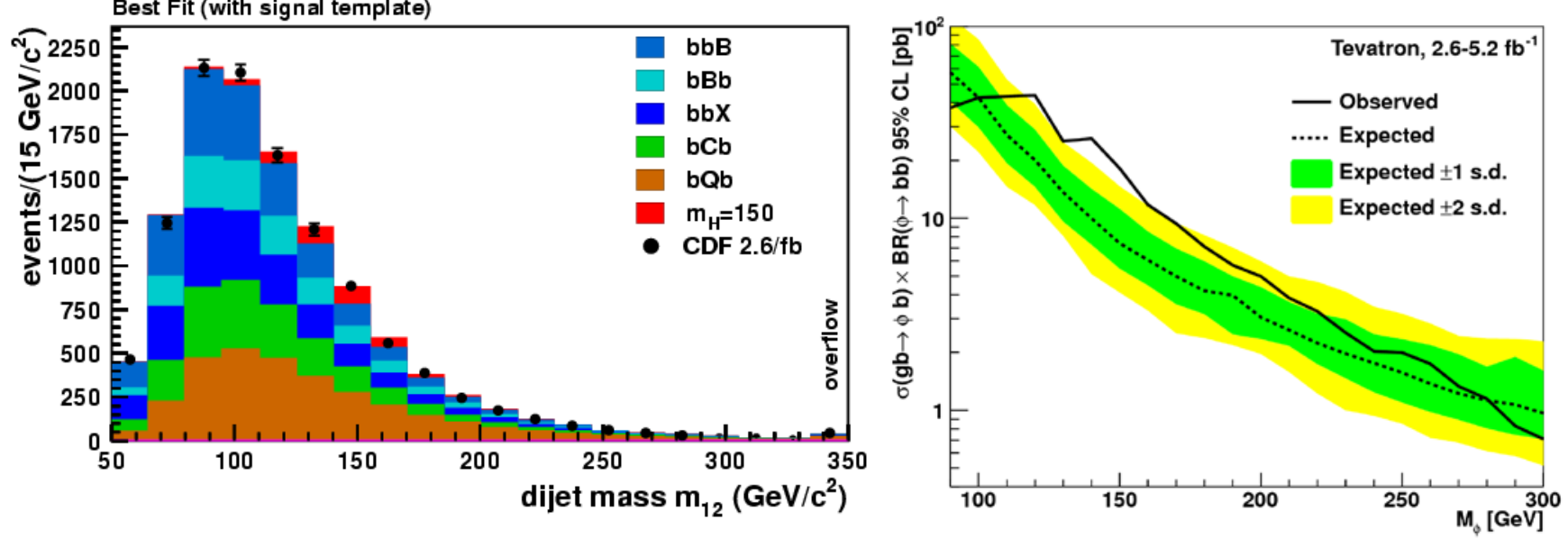

5-Sep-2013

R. Mankel; Higgs Searches Beyond the SM
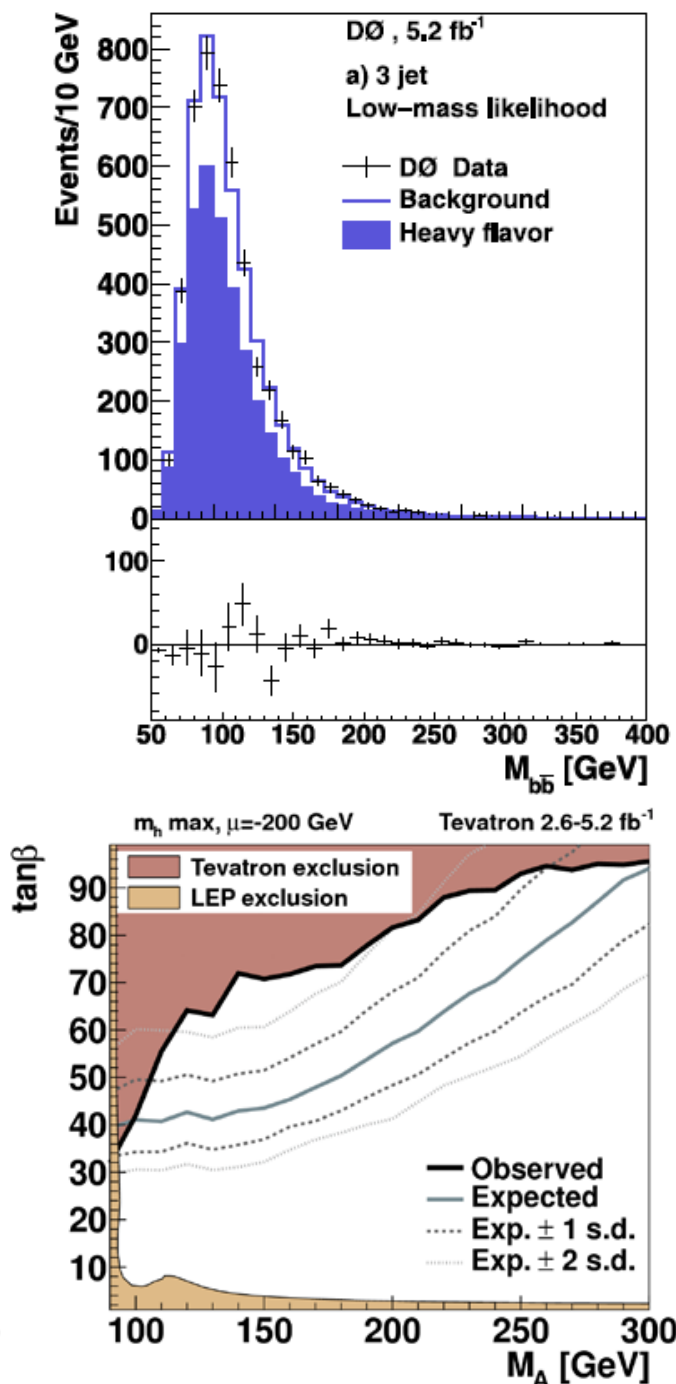

15 
- CMS analysis searches in all-hadronic (shown) and semi-leptonic signatures (see backup)

- All-hadronic analyses inspired by CDF method

$\rightarrow$ Background-only fit with shapes

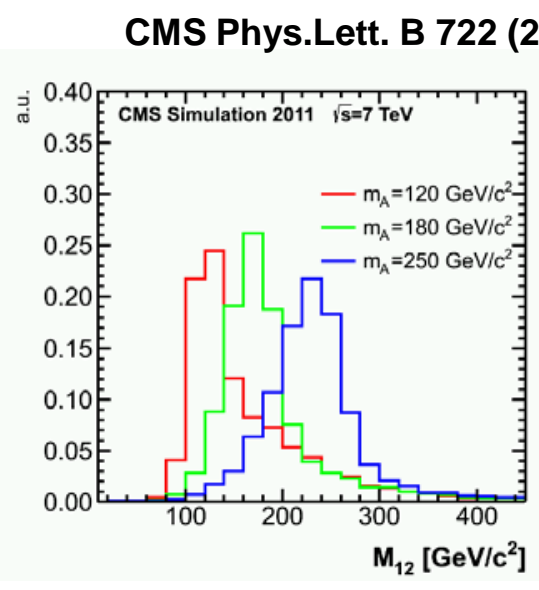
determined from double-btag sample gives excellent agreement with triplebtag data

- No signal observed

- First measurement of this kind at the LHC
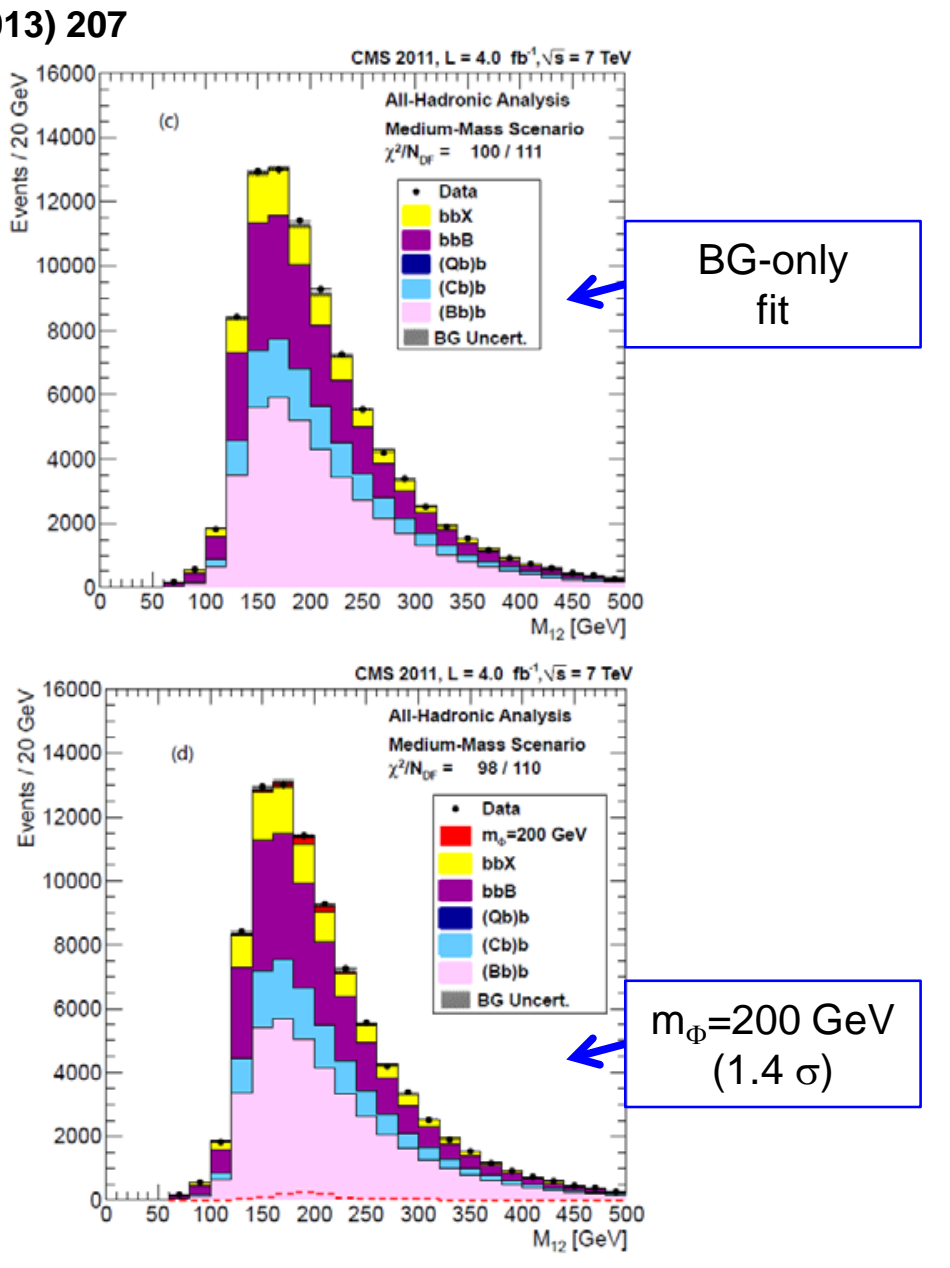


\section{$\Phi \rightarrow$ bb Search: Combination of CMS Analyses \& Comparison with Tevatron}

CMS Phys.Lett. B 722 (2013) 207
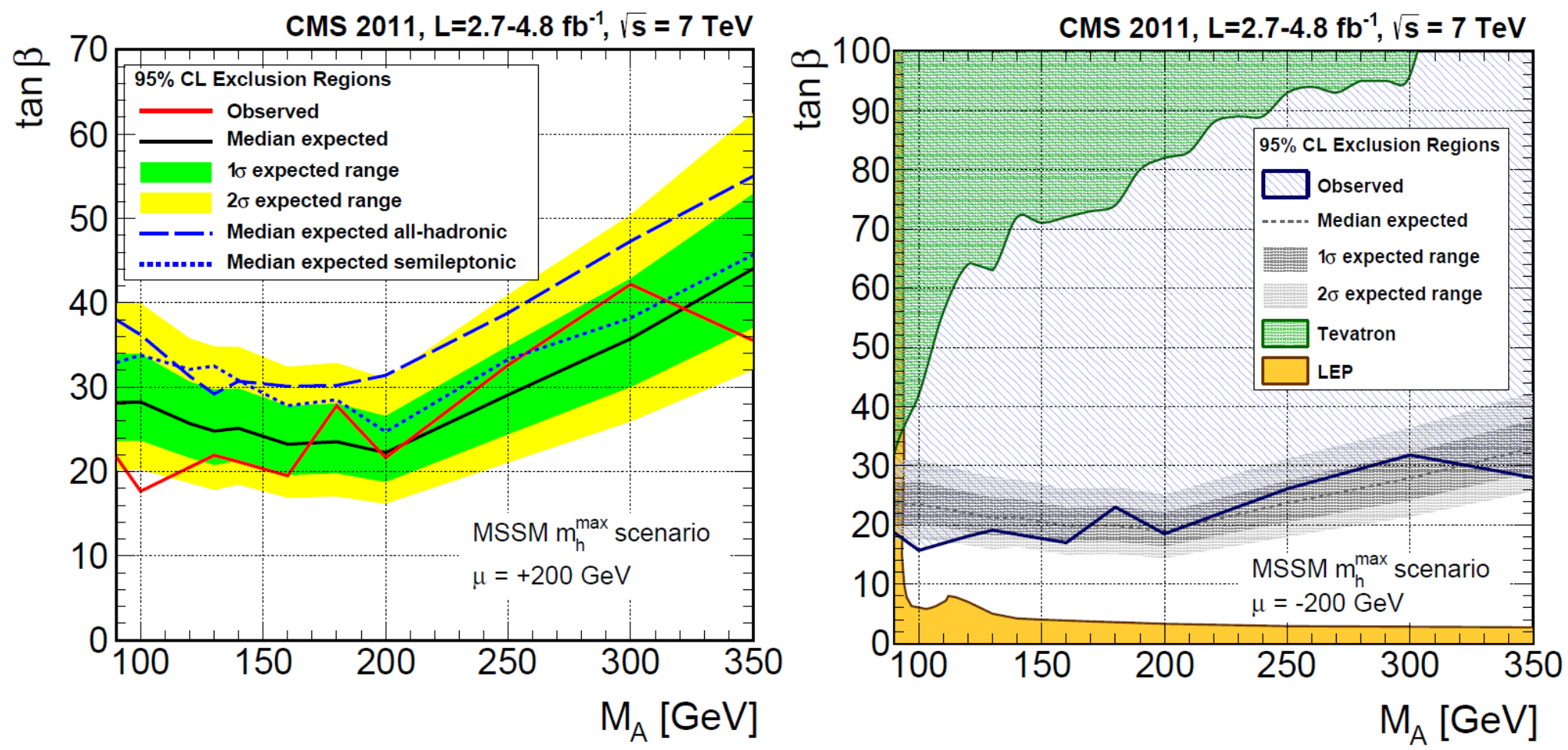

- For comparison with Tevatron, CMS results also given for $\mu=-200 \mathrm{GeV}$ (right)

$\rightarrow$ Already with 2011 data, CMS has significantly higher sensitivity

$\rightarrow$ CMS does not confirm $\sim 2 \sigma$-level excesses seen by CDF + D0 


\section{$\mathbf{H}^{ \pm} \operatorname{Search}\left(\mathbf{H}^{+} \rightarrow \tau v_{\tau}\right.$ Mode $)$}

- $\mathrm{H}^{+} \rightarrow \tau v_{\tau}$ dominant decay mode for tan $\beta>5$ and $\mathrm{m}\left(\mathrm{H}^{+}\right)<\mathrm{m}_{\mathrm{t}}$

ATLAS CONF-2013-090

- Main production modes:

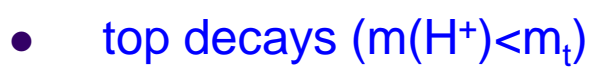
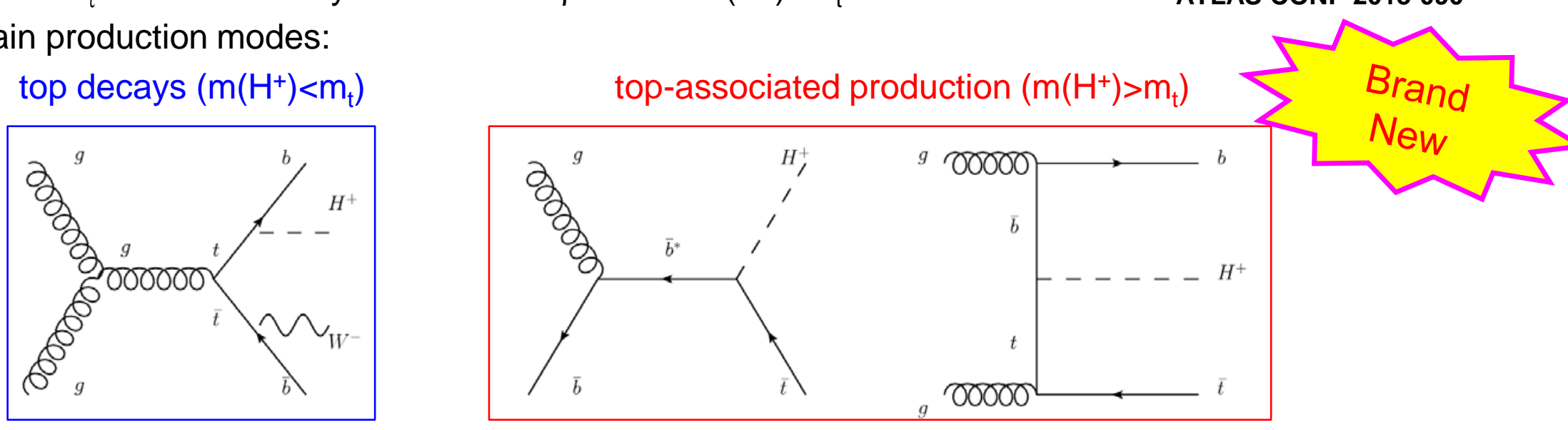

- Fully hadronic final states (ATLAS):

- veto on other leptons

- 3-4 jets ( $\geq 1$ b-tagged)

- large MET

- Discriminating variable $\mathrm{m}_{\mathrm{T}}$

(= transverse invariant mass of $\tau+$ MET)

- Backgrounds: $t \bar{t}$, single-top, W/Z+jets, di-bosons, QCD

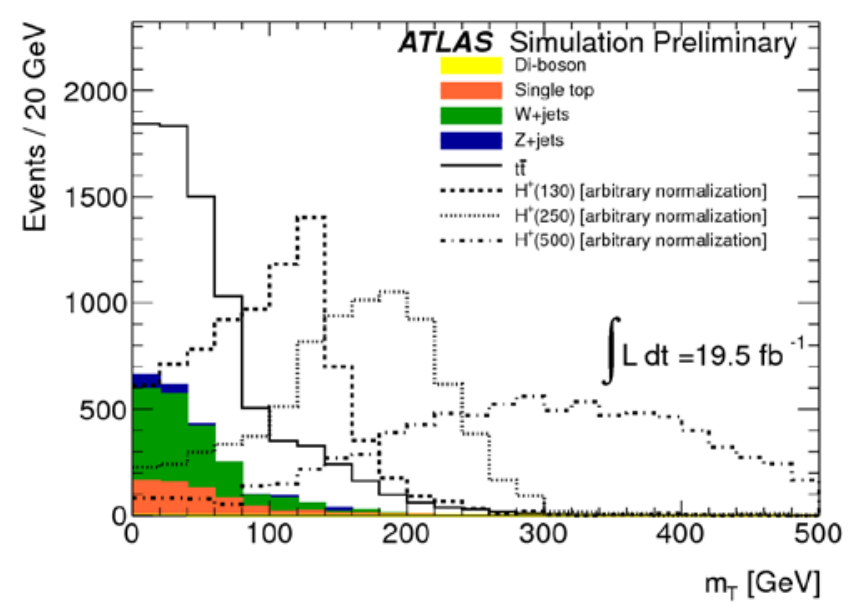




\section{CMS \\ $H^{+} \rightarrow \tau v_{\tau} \operatorname{Search}($ cont'd)}
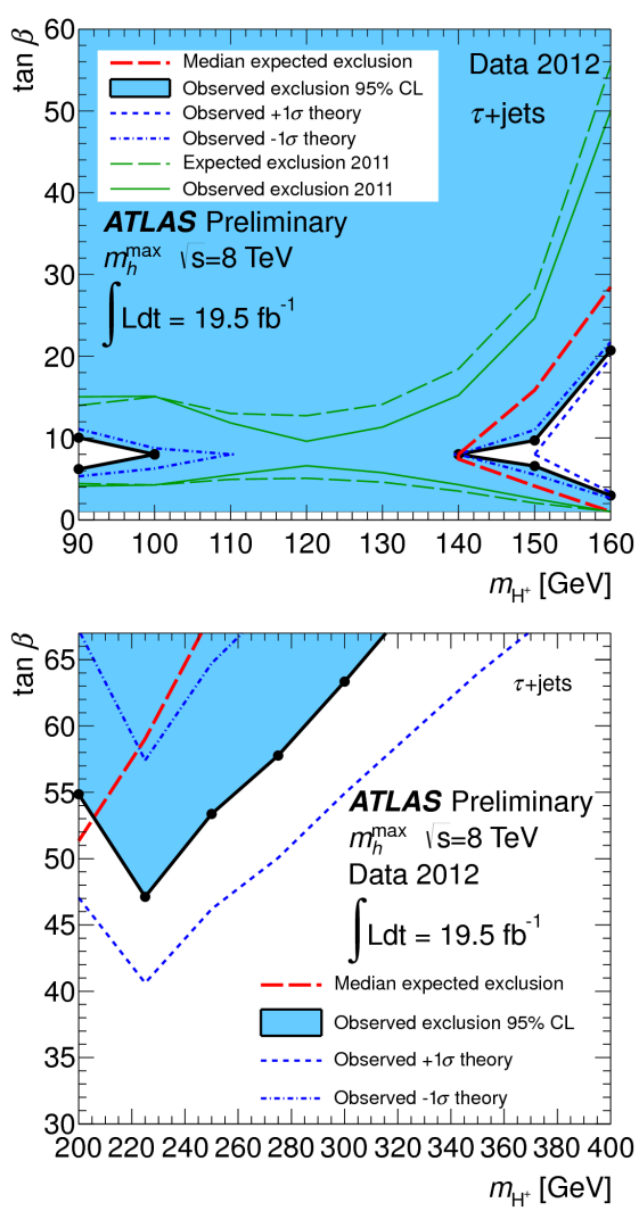

ATLAS CONF-2013-090

- Full 2012 dataset used

- Mass ranges 90-160, 180-600 GeV covered

No evidence for $\mathrm{H}^{+}$ found

- At low $\mathrm{m}\left(\mathrm{H}^{+}\right)$, large parts of MSSM parameter space excluded 
- For $\tan \beta<1, H^{ \pm} \rightarrow c \bar{s}$ becomes the dominant decay mode ( $70 \%$ for $\left.m_{H^{ \pm}} \sim 110 \mathrm{GeV}\right)$

- First investigation of this process by CDF and D0 at the Tevatron collider

$\rightarrow$ no indication for $\mathrm{H}^{+}$signal

$\rightarrow$ upper limits on $\mathrm{B}\left(\mathrm{t} \rightarrow \mathrm{H}^{+}\right.$b) around $10-30 \%$, assuming $\mathrm{B}\left(\mathrm{H}^{+} \rightarrow \boldsymbol{c} \overline{\boldsymbol{s}}\right)=100 \%$

- In the mean time, has been measured by ATLAS at the LHC

CDF, Phys. Rev. Lett. 103 (2009) 101803.

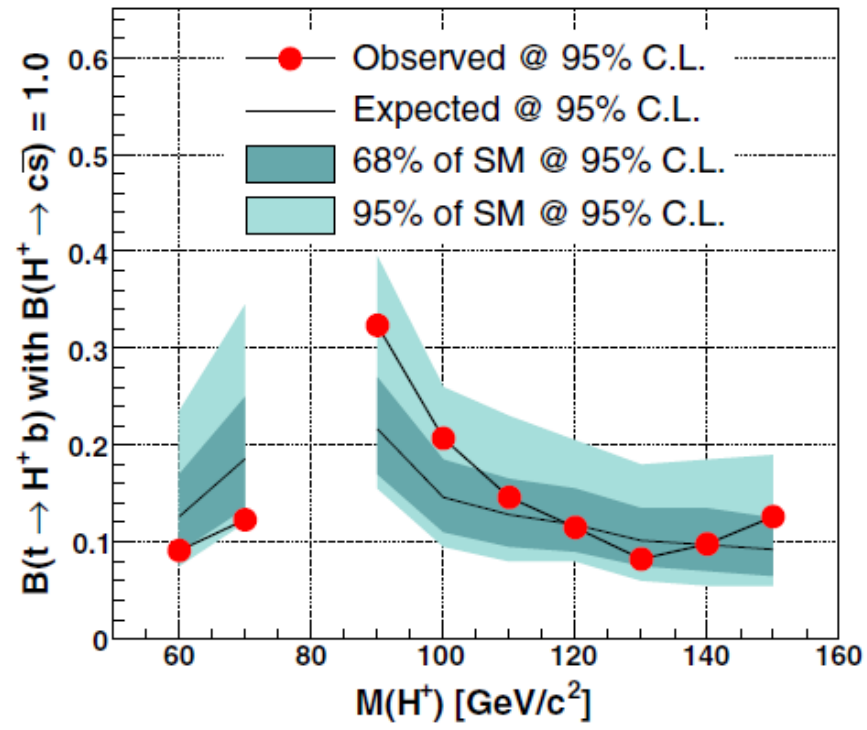

D0, Phys. Lett. B 682 (2009) 278.

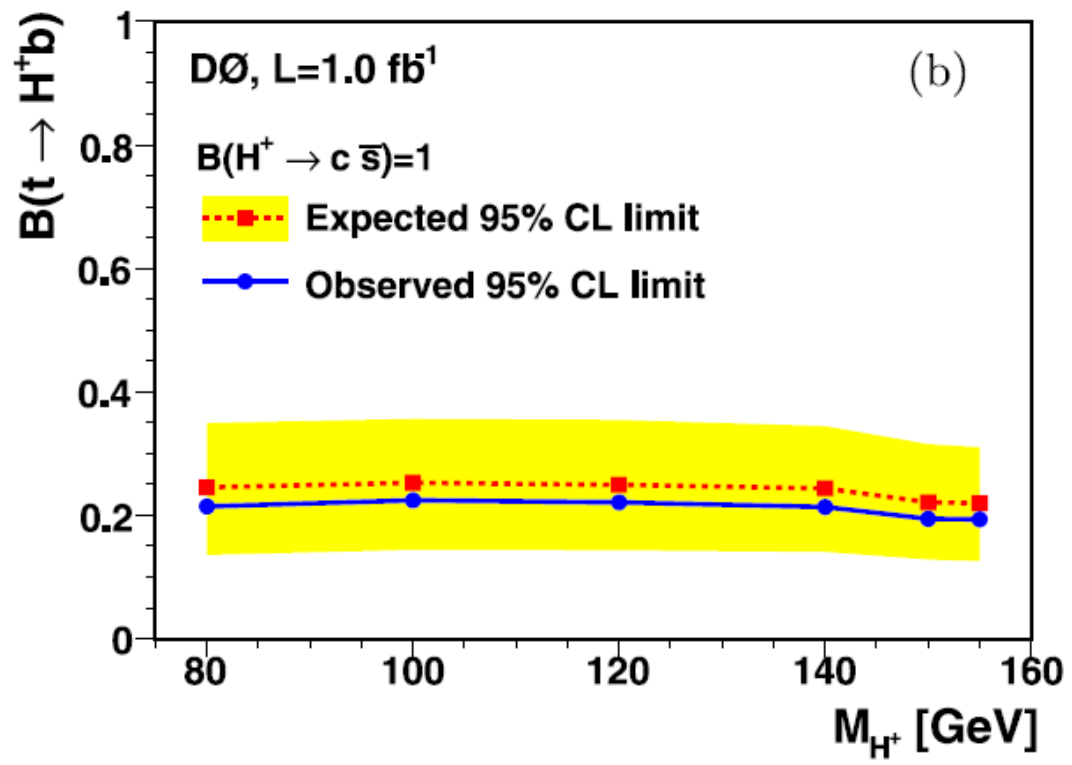


- Same topology as $t \bar{t}$ decays in lepton + jets channel

- Search for second peak in the di-jet mass distribution

- Event selection:

- isolated lepton, 4 jets (two with b-tag)

- MET

- Kinematic fit for mass reconstruction is essential

$\rightarrow$ Significant improvement of separation between standard ttbar background and signal $\left(\sigma_{\mathrm{m}} \sim 12 \mathrm{GeV}\right)$

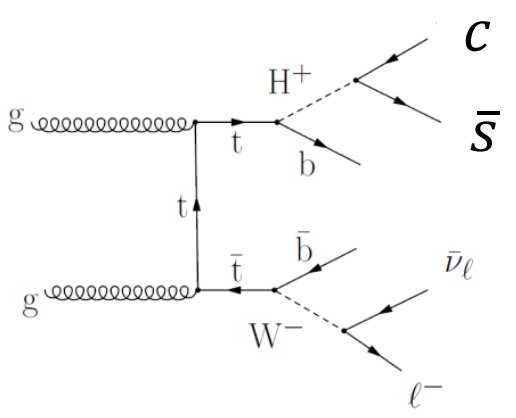

ATLAS Collab., Eur. Phys. J. C 73 (2013) 2465.
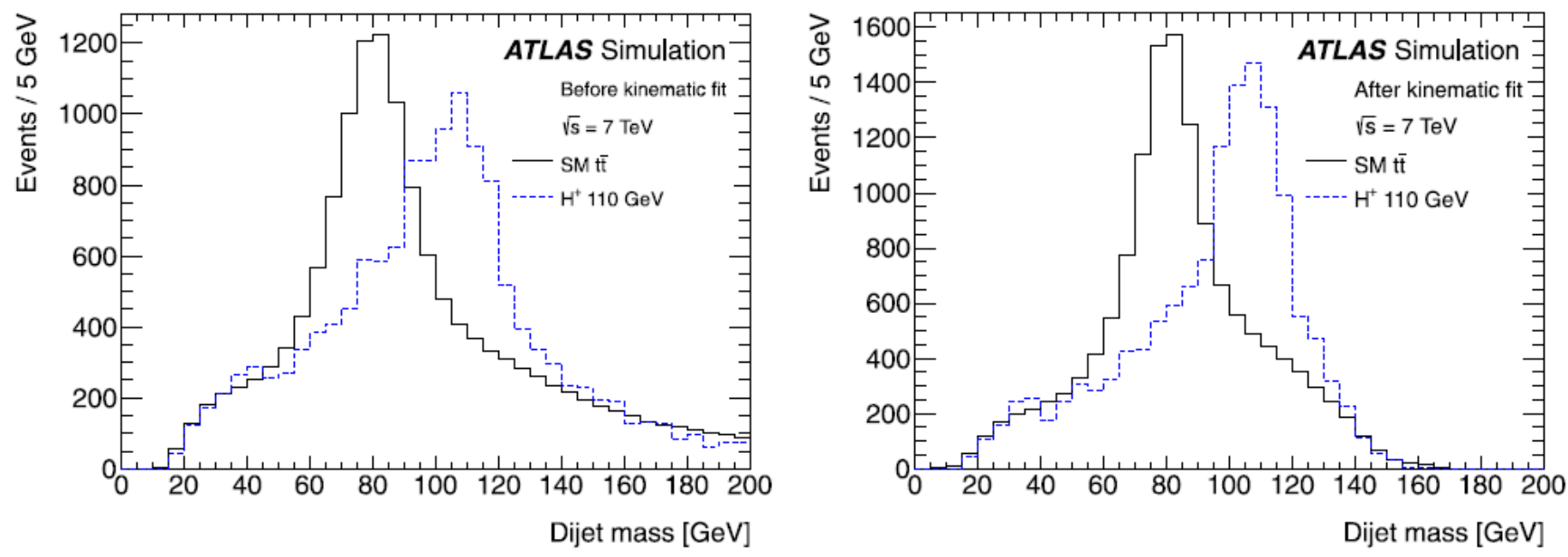
ATLAS Collab., Eur. Phys. J. C 73 (2013) 2465.

- Main backgrounds:

- $\operatorname{SM} t \bar{t}$

- QCD multi-jet (data-driven, shape from semi-isolated lepton control region)

- $\quad W / Z+j e t s$, single top

$\rightarrow$ Observed mass spectrum well described by background estimation

- $\quad$ no indication for $\mathrm{H}^{+}$signal

$\rightarrow$ Observed limits between 1-5 \%

$\rightarrow$ Most stringent results to date in $c \overline{\boldsymbol{s}}$ channel
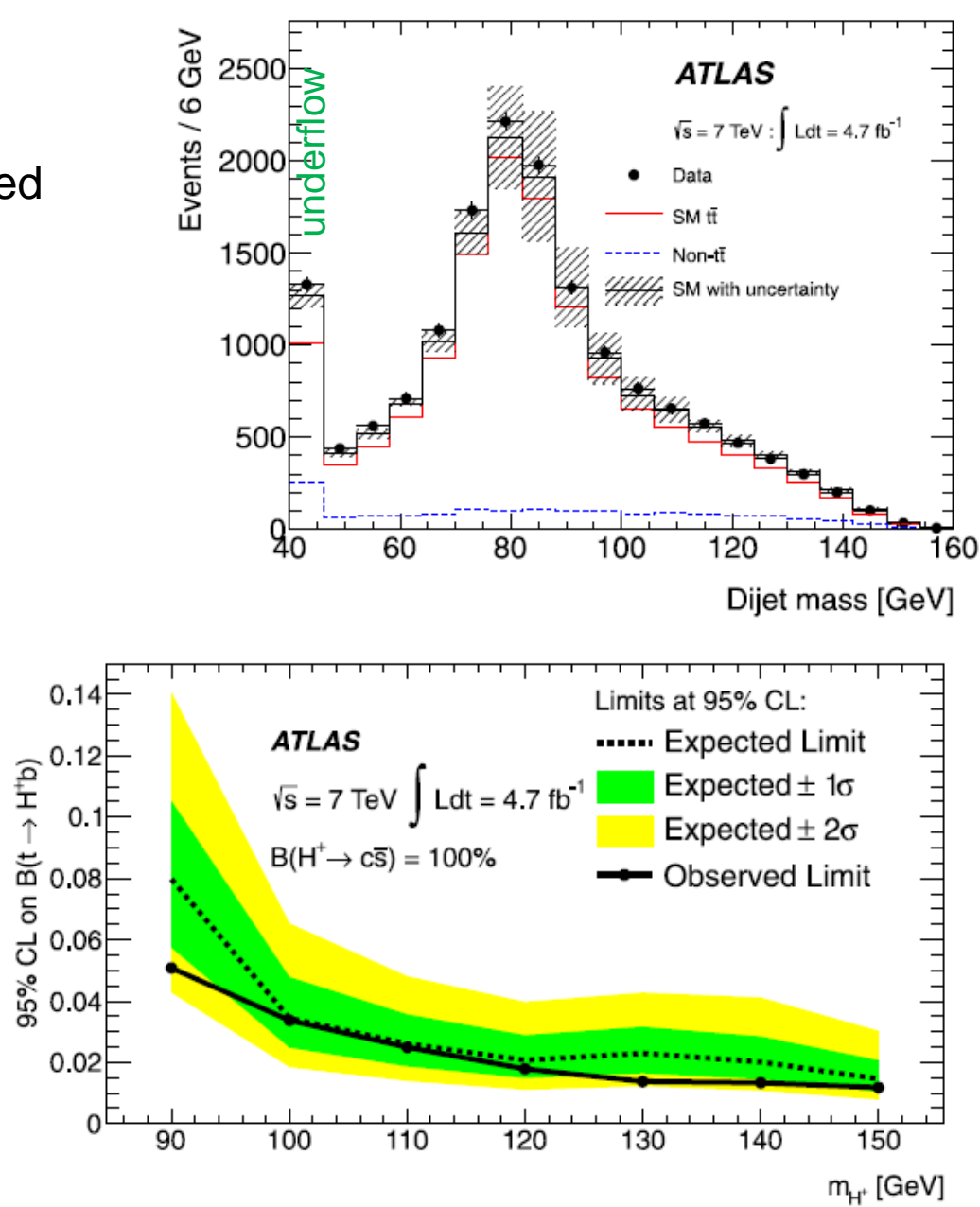

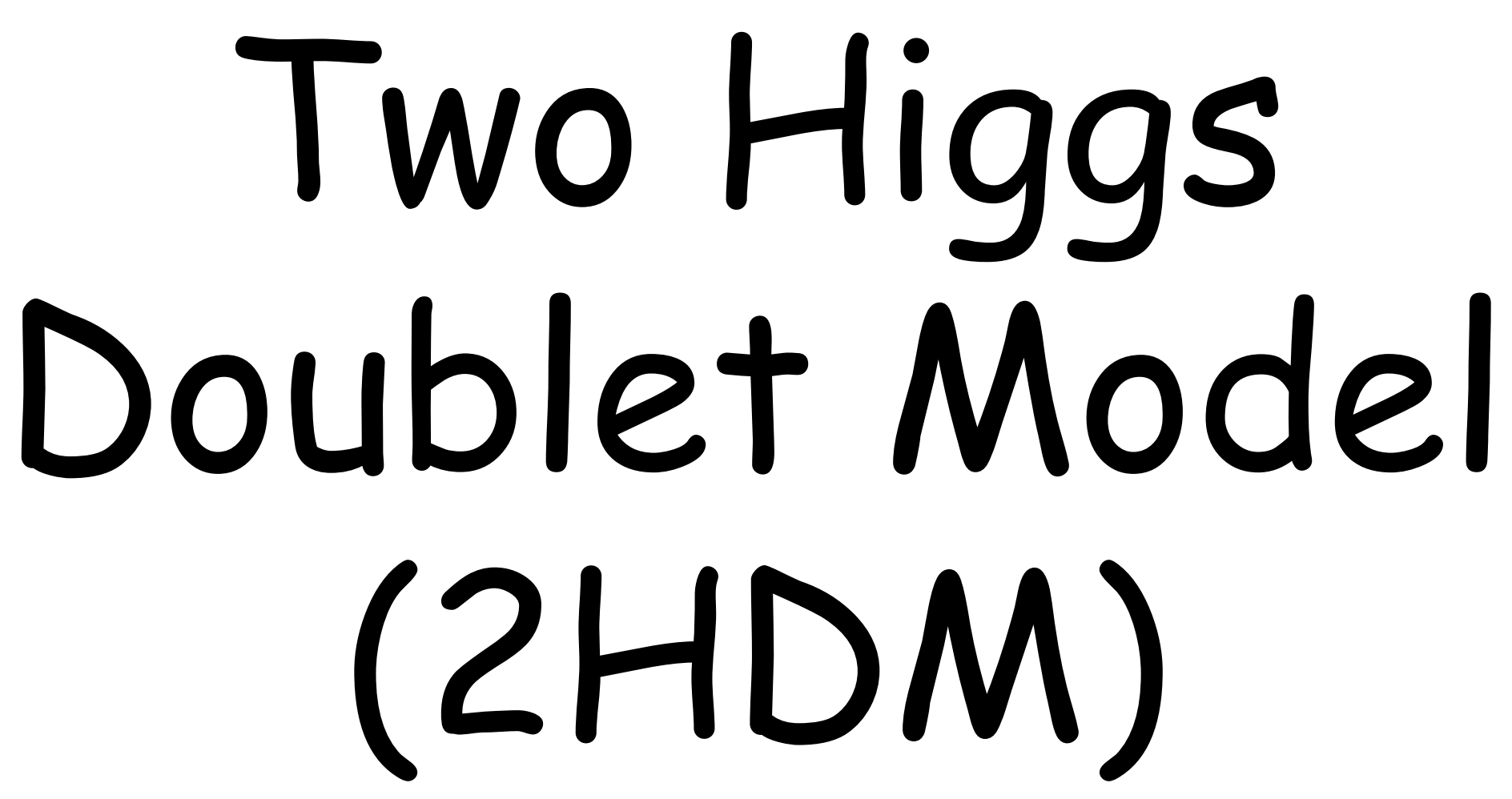
- Phenomenological approach: simple extension of SM Higgs sector by second complex Higgs doublet

- five Higgs bosons: $\mathrm{h}, \mathrm{H}, \mathrm{A}, \mathrm{H}^{ \pm}$

- can accommodate CP violation (as opposed to MSSM at tree level)

$\rightarrow$ possible explanation of baryon asymmetry in universe?

- Examples of 2HDM models with natural flavor conservation:

- Type I: all quarks couple only to one Higgs doublet

- Type-II: up-type quarks $(\mathrm{Q}=+2 / 3)$ couple to one, down-type quarks

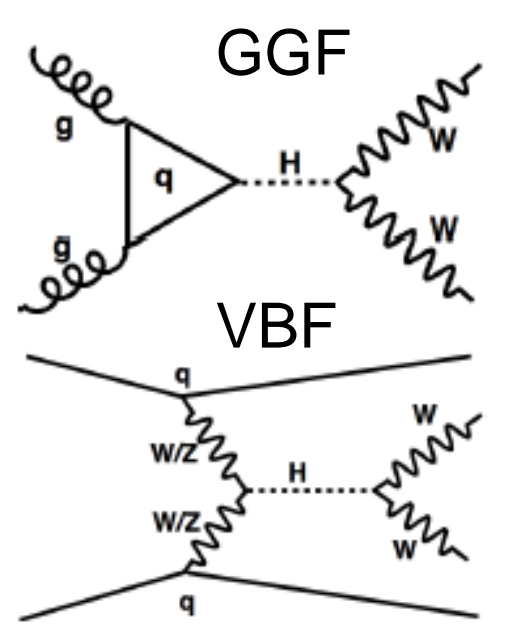
$(\mathrm{Q}=-1 / 3)$ couple to the other Higgs doublet

- $\quad \tan \beta$ : ratio of VEVs. $\quad \alpha$ : scalar mixing angle

- ATLAS analysis: $\mathrm{h} / \mathrm{H} \rightarrow \mathrm{WW}^{(*)} \rightarrow \mathrm{e} v \mu \nu$, assume $\mathrm{m}_{\mathrm{h}}=125 \mathrm{GeV}$

- pseudoscalar A does not decay to W pairs

- exactly 2 leptons of opposite charge, $\mathrm{E}_{T, \text { rel }}$ miss

- gluon-gluon fusion (GGF) selection: zero jets

- vector-boson fusion (VBF) selection: two jets

- neural network combines kinematic variables to enhance S/B

- trained for each mass point

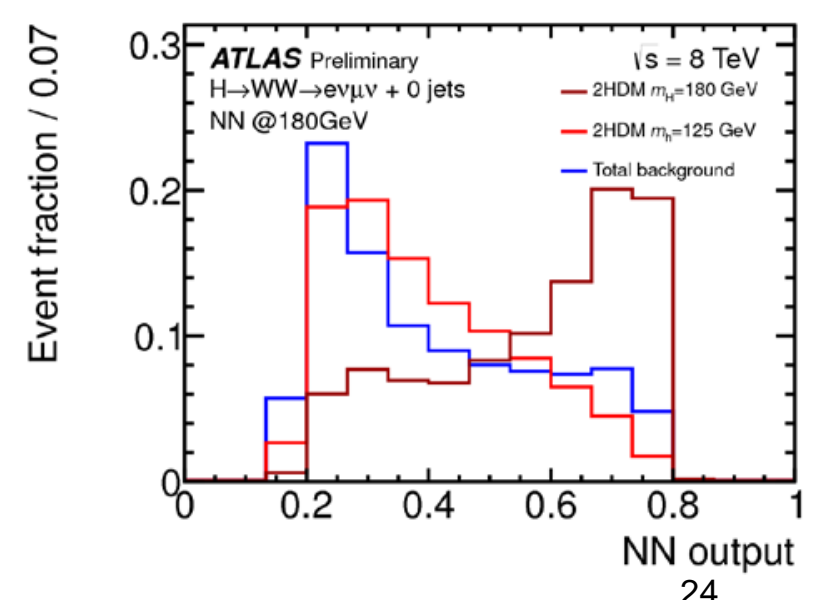




\section{HDM Higgs Search: Results}

- $\quad \mathrm{H}(125)$ treated as "background"

$\rightarrow$ No indication of a signal (would appear at large NN output)

ATLAS CONF-2013-027
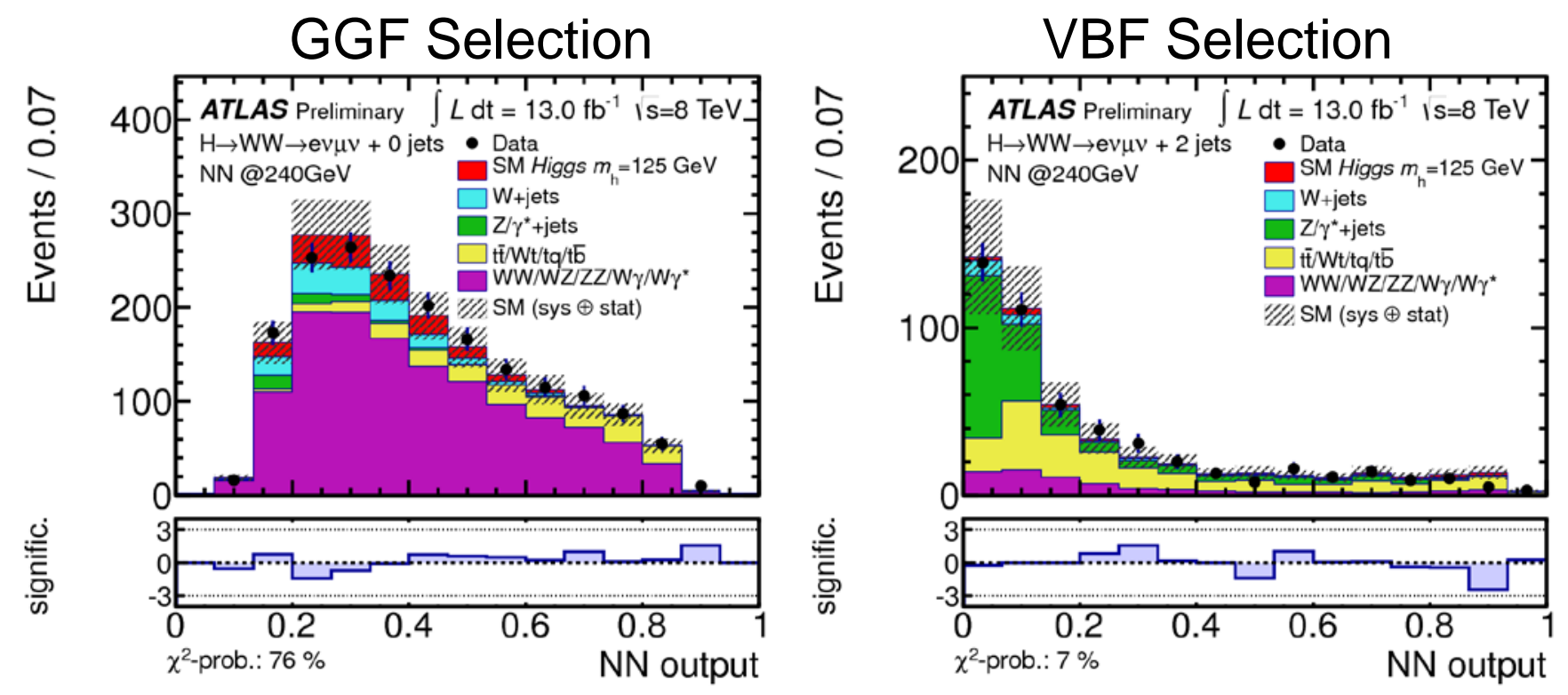


\section{HDM Higgs Search: Results}

ATLAS CONF-2013-027

$\tan \beta=1$
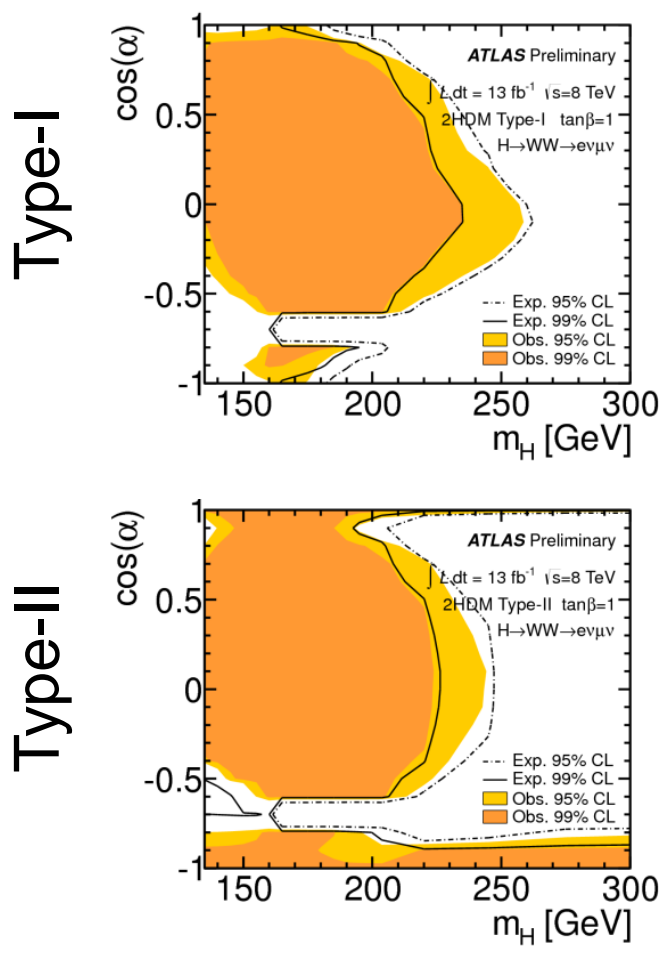

$\tan \beta=20$
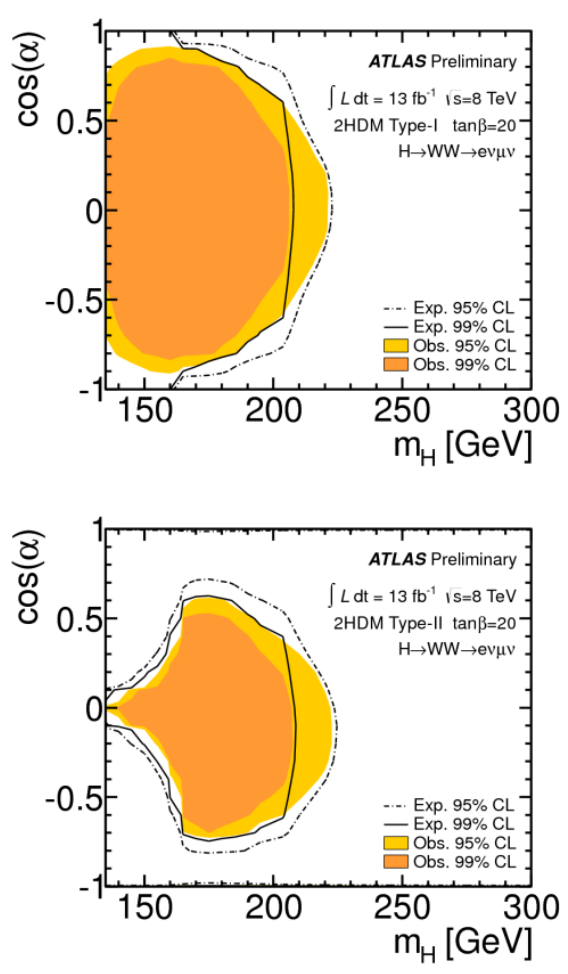

- Interpretation: exclusion contours in the $\cos \alpha-m_{H}$ plane for different values of $\tan \beta$

- different results for Type-I and Type-II 2HDM models

$\rightarrow$ For low masses (<200 GeV), significant parts of the $\cos \alpha$ range are excluded 
Phys.Rev.Lett. 110 (2013) 121801

- Search for a heavy scalar $\mathrm{H}^{0}$ with a cascade decay:

- $\mathrm{H}^{0} \rightarrow \mathrm{H}^{+} \mathrm{W}^{-} \rightarrow\left(\mathrm{h}^{0} \mathrm{~W}^{+}\right) \mathrm{W}^{-} \rightarrow(b \bar{b}) \mathrm{W}^{+} \mathrm{W}^{-}$

- one $W$ decaying leptonically

- final state similar to ttbar events

- 1 lepton, $\geq 4$ jets, $\geq 1$ b-tags, MET>20 GeV

- Dominant backgrounds:

- $t \bar{t}$ production modeled by MC (PYTHIA)

- $W+$ jets background modeled with

ALPGEN/PYTHIA
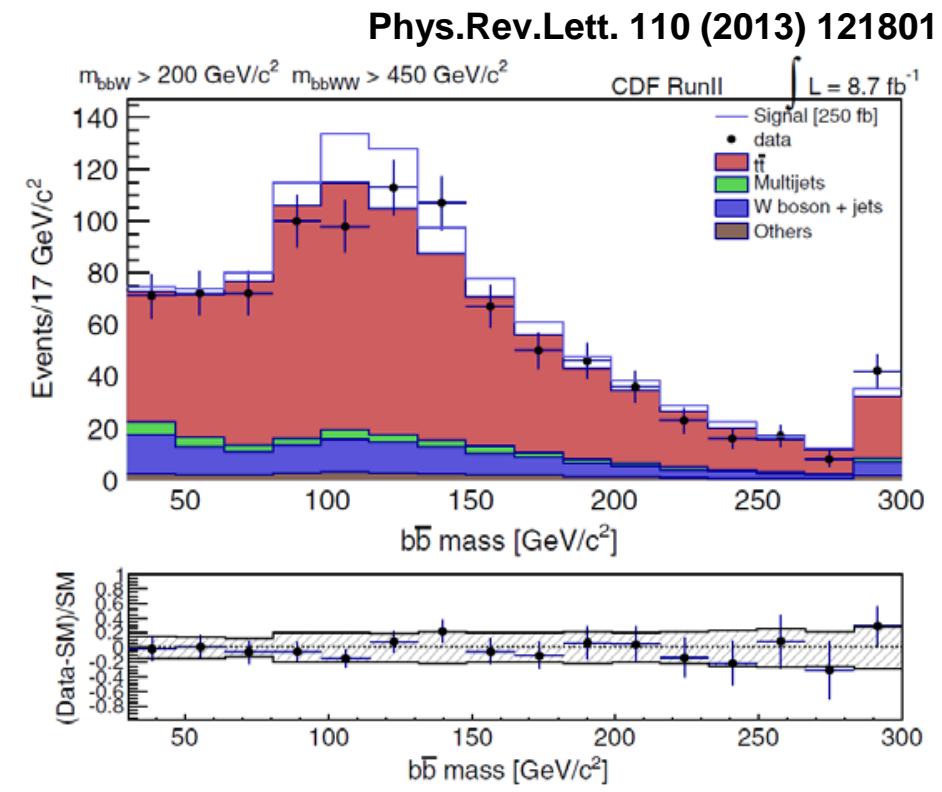

- Reconstruct $1 \mathrm{~W}$ from lepton+MET and the other from jet pair with matching mass

- search signal in $\mathrm{m}_{\mathrm{bb}}$

- Cross section upper limits obtained scanning the space of $\mathrm{H}^{ \pm}$and $\mathrm{H}^{0}$ masses

- assume $\mathrm{B}\left(\mathrm{H}^{0} \rightarrow \mathrm{H}^{+} \mathrm{W}^{-}\right)=\mathrm{B}\left(\mathrm{H}^{+} \rightarrow \mathrm{h}^{0} \mathrm{~W}^{+}\right)=100 \%$

$\Rightarrow \quad$ limits range between 1.3-0.015 pb

$\rightarrow \quad$ first measurement of this kind

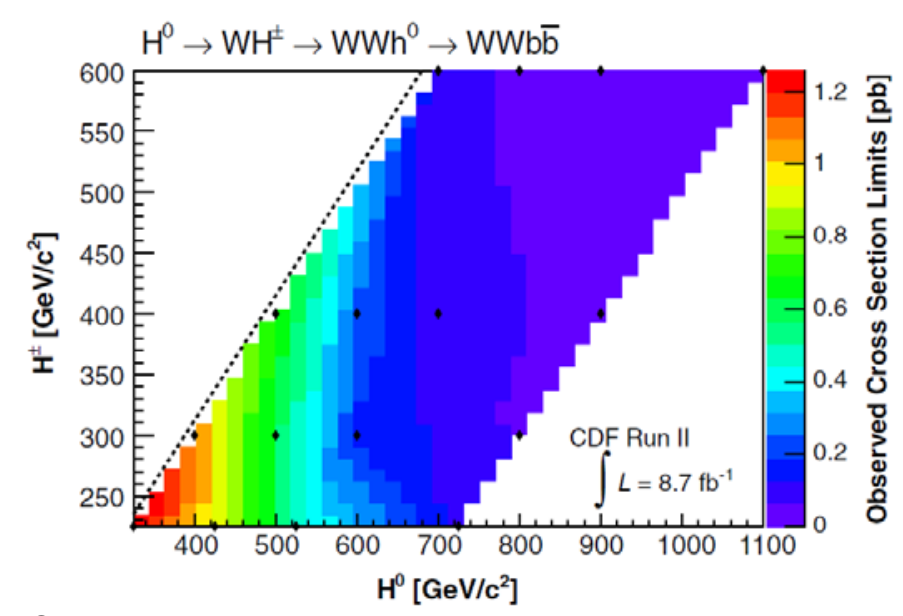


NMSSM Higgs Bosons 


\section{The NMSSM Higgs Sector}

- Two complex Higgs doublets + additional scalar field

- Physical states are mixtures: $h_{1}, h_{2}, h_{3}, \quad a_{1}, a_{2}, h^{ \pm}$

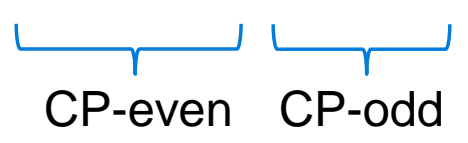

$\rightarrow$ Requires less fine tuning for Higgs mass, solves " $\mu$ problem" of MSSM

$\rightarrow$ Rich phenomenology

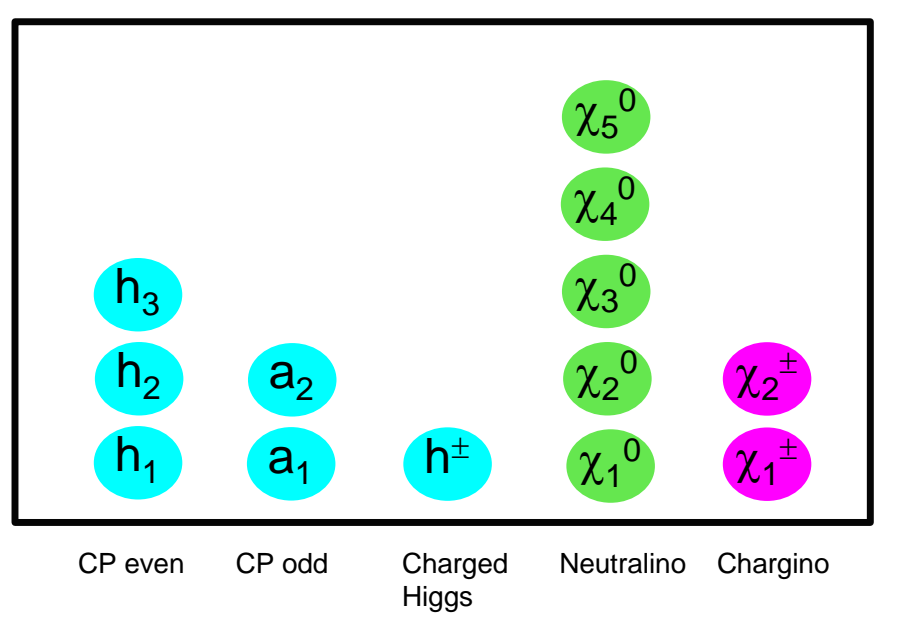


CMS PAS HIG-13-010

- Search for a non-standard Higgs decay into two very light bosons, resulting in two boosted pairs of muons

- NMSSM interpretation: $h_{1,2} \rightarrow a_{1} a_{1} \rightarrow(\mu \mu)(\mu \mu)$

- $\quad$ either $h_{1}$ or $h_{2}$ could correspond to observed $H(125)$

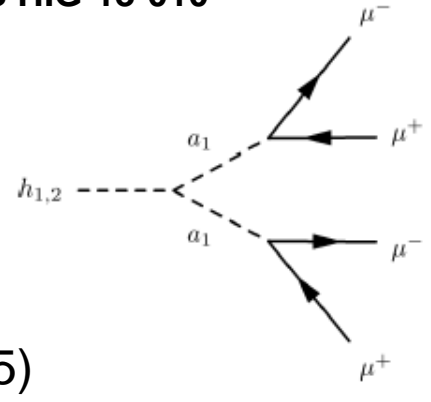

- $a_{1}$ is a new light CP-odd Higgs boson $\left(m<2 m_{\tau}\right)$

- Dark SUSY interpretation: $\mathrm{h} \rightarrow 2 \mathrm{n}_{1} \rightarrow 2 \mathrm{n}_{\mathrm{D}}+2 \gamma_{\mathrm{D}} \rightarrow 2 \mathrm{n}_{\mathrm{D}}+(\mu \mu)(\mu \mu)$

- models motivated by excesses in positron spectra observed by satellite experiments

- cold dark matter with a mass scale of $\sim 1 \mathrm{TeV}$

- $\mathrm{n}_{1}$ is lightest visible neutralino, $\mathrm{n}_{\mathrm{D}}$ is light dark fermion, and $\gamma_{\mathrm{D}}$ light (massive) dark photon that weakly couples to SM particles

- Search for events with two isolated, boosted muon pairs

- consider $0.25<\mathrm{m}_{\mathrm{a}}<3.55 \mathrm{GeV}$ and $\mathrm{m}_{\mathrm{h}}>86 \mathrm{GeV}$

- Signal region: $\mathrm{m}_{\mu \mu 1} \approx \mathrm{m}_{\mu \mu 2}$

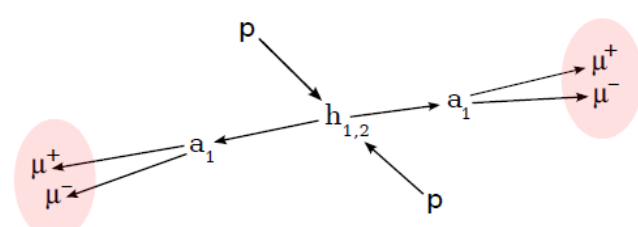

- Main backgrounds:

- direct double-J/ $\psi$ production

- $\quad b \bar{b}$ production with subsequent di-muon decays (double-semileptonic or resonances) 


\section{$h_{1,2} \rightarrow a a \rightarrow(\mu \mu)(\mu \mu):$ Results}

- $\quad b \bar{b}$ background from bb-enriched control sample, double-J/ $\psi$ production from PYTHIA

$\rightarrow \quad 8$ events observed in off-diagonal sideband

$\rightarrow$ After unblinding, only 1 event is observed in the diagonal signal region

$\rightarrow \quad$ expected background: $3.8 \pm 2.1$ events

CMS PAS HIG-13-010

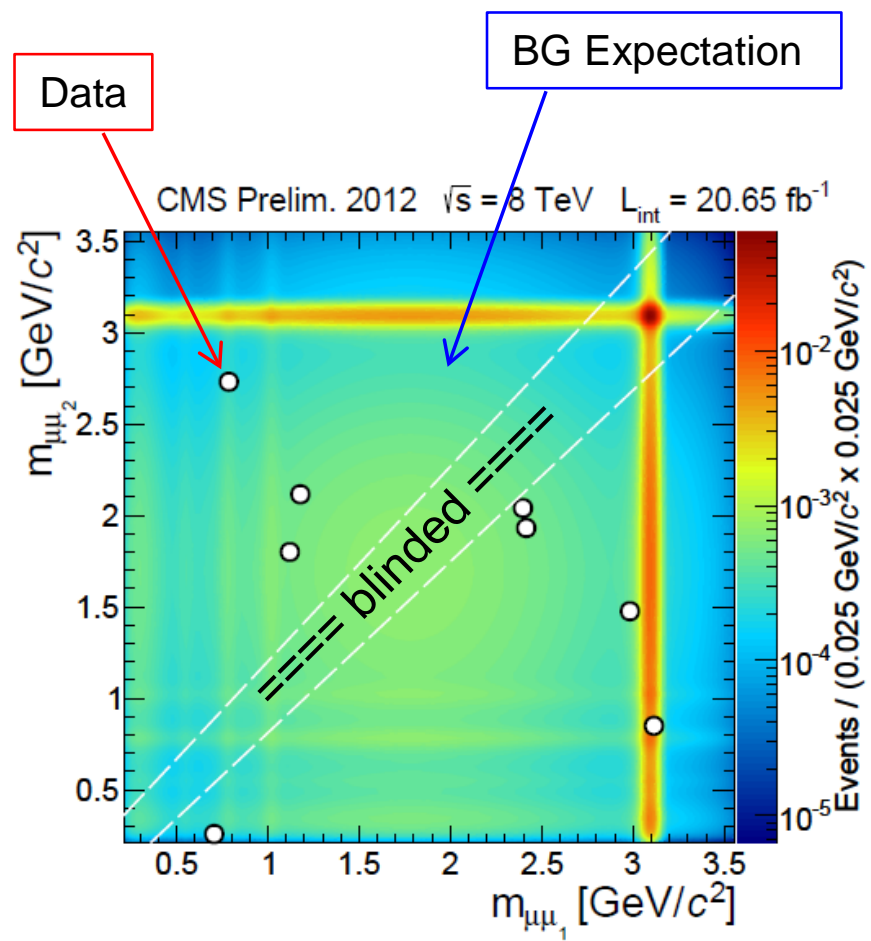

after unblinding (signal region):

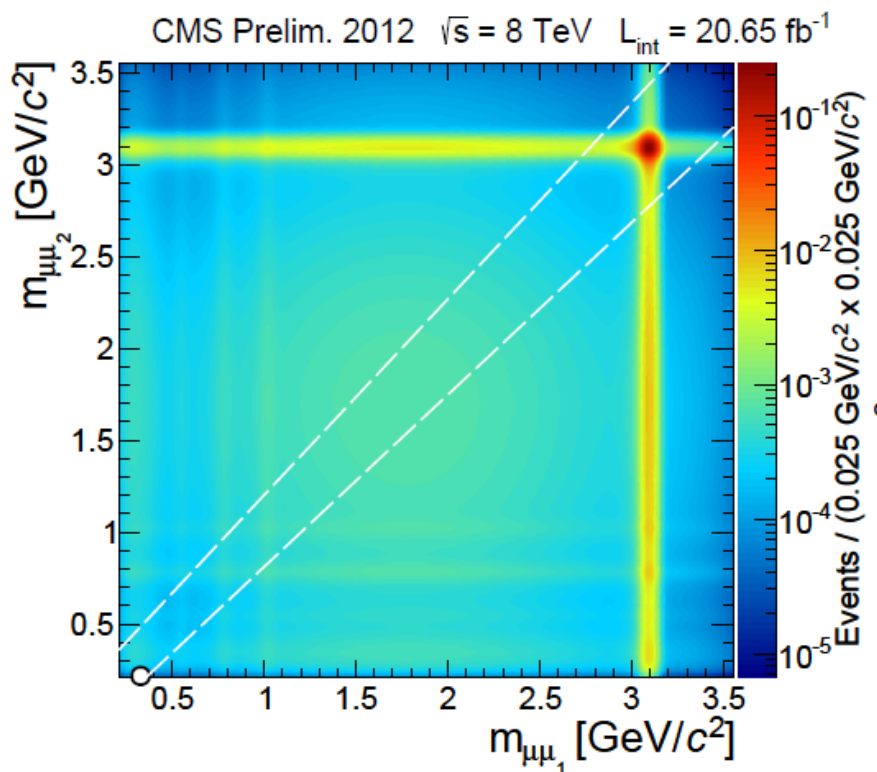



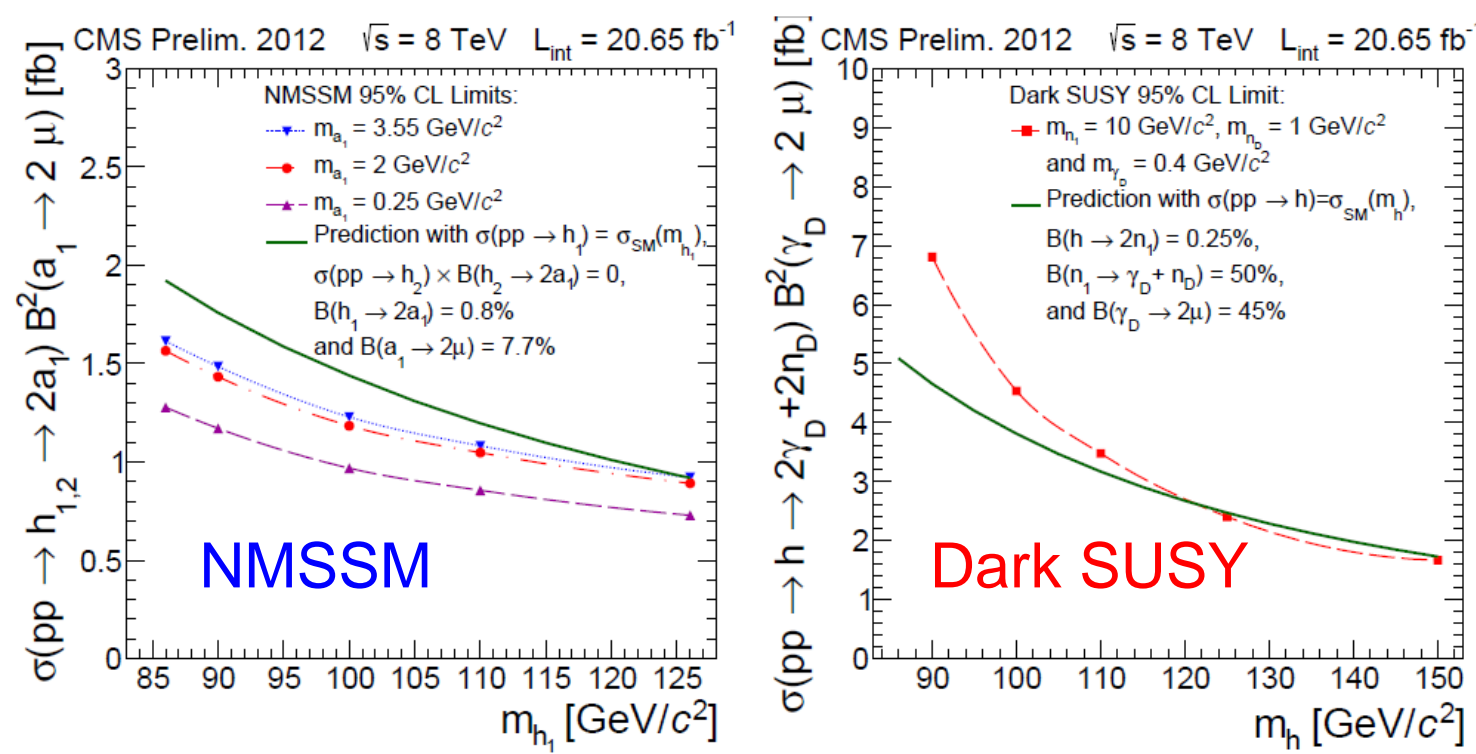

- Results are interpreted in context of NMSSM and dark-SUSY benchmark models

- signal efficiencies depend on assumptions for either $m_{a}$ or $m_{h}$

$\rightarrow$ NMSSM: upper limits vs $m_{h 1}$ and $m_{\mathrm{a} 1}$

- $\quad \mathrm{m}_{\mathrm{h} 2}$ unrestricted $\rightarrow$ conservative assumption on efficiency

- for NMSSM prediction, assume that only $\mathrm{h}_{1}$ decays into $2 \mathrm{a}_{1}$

$\rightarrow$ Dark SUSY: upper limits vs $m_{h}$

$\rightarrow$ Best experimental limits in this signature 


\section{CMS/ H(125) Decays to Light NMSSM Pseudo- Scalars}

- If very light NMSSM CP-odd Higgs bosons exist, they might be observed in the decay

$$
\mathrm{H}(125) \rightarrow \mathrm{a} \mathrm{a} \rightarrow(\gamma \gamma)(\gamma \gamma)
$$

- Here: assume $m_{a}=100-400 \mathrm{MeV}$

- di-photon system would be highly boosted. Potential background for SM $\mathrm{H} \rightarrow \gamma \gamma$ analysis

- Analysis similar to $\mathrm{SM} \mathrm{H} \rightarrow \gamma \gamma$

- additional selection based on shower shape variables \& calorimetric isolation

$\rightarrow$ No signal observed

- upper limits of $\sigma^{*} \mathrm{BR}(\mathrm{H} \rightarrow \mathrm{aa} \rightarrow(\gamma \gamma)(\gamma \gamma))$ in 0.1-0.2 pb range

ATLAS CONF-2012-079
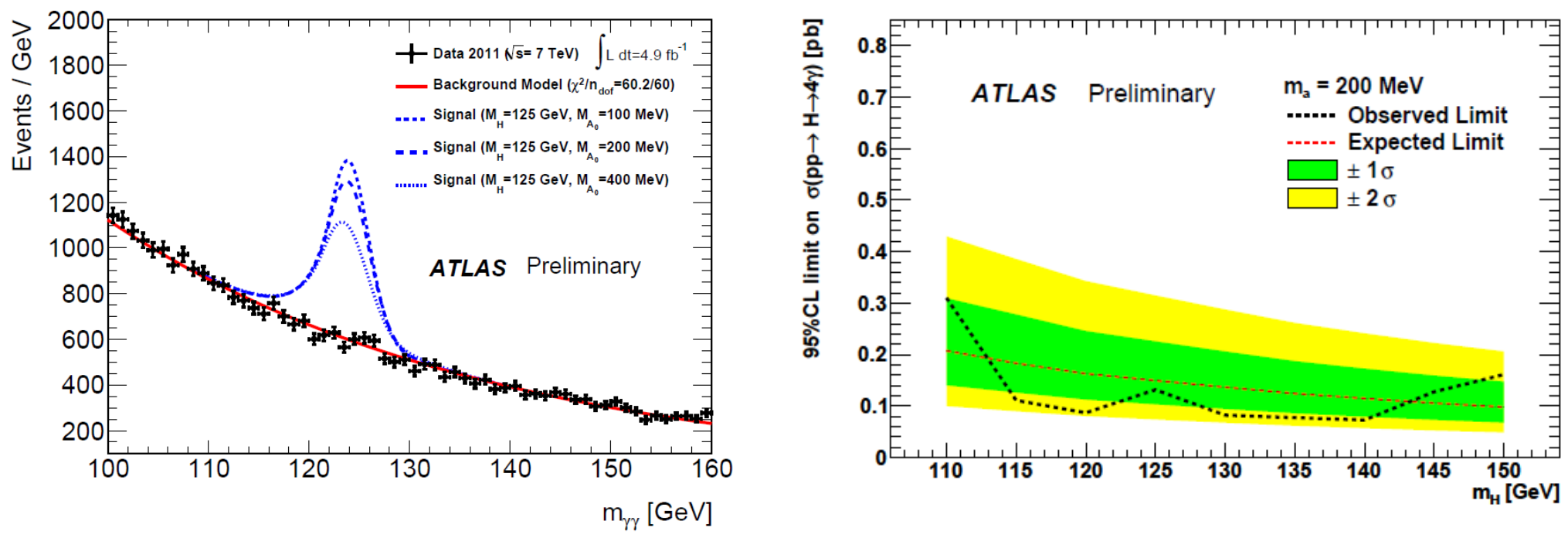


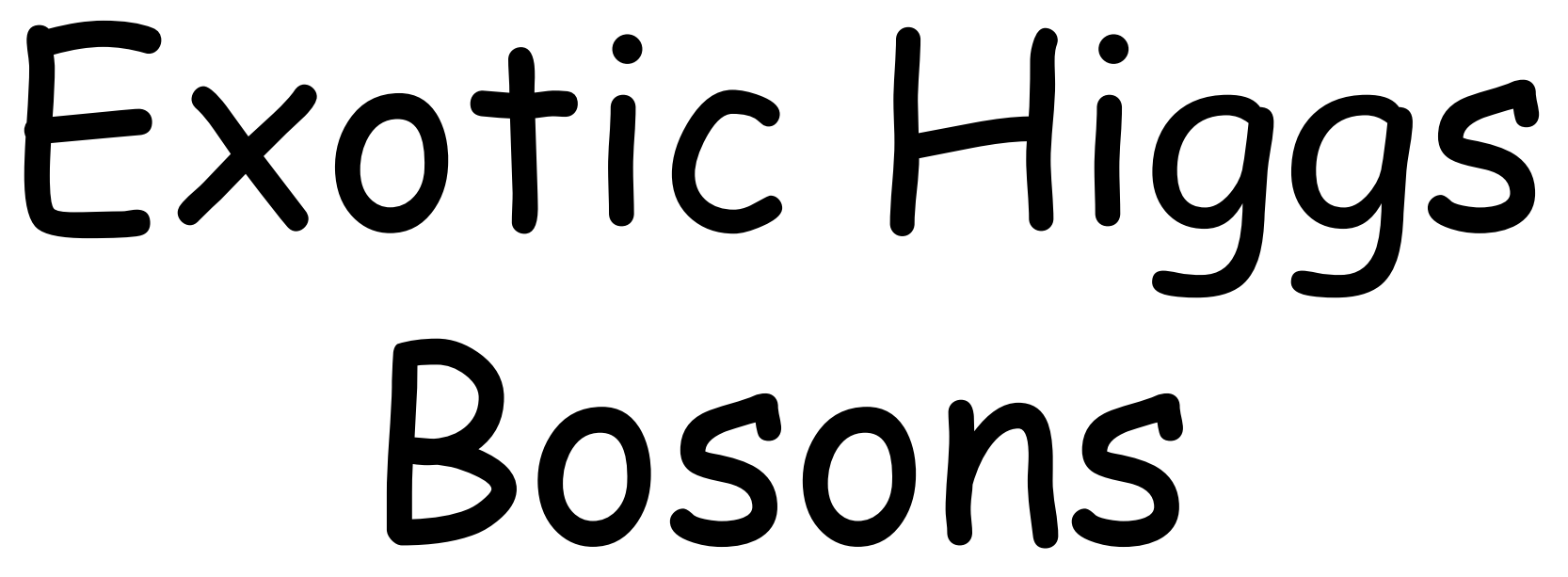 \\ Exotic Higgs Bosons}


- If the Higgs would decay with a significant fraction to invisible particles, this might be detectable in associated production with a $Z$ boson

$\rightarrow$ look for events with $\left.Z \rightarrow I^{+}\right|^{-}$plus missing $E_{T}$, and little else

- Main backgrounds:

- $\quad Z Z \rightarrow\left\|_{v v}, Z W \rightarrow\right\|\left\|_{v}, W W \rightarrow I_{v}\right\|_{v}$

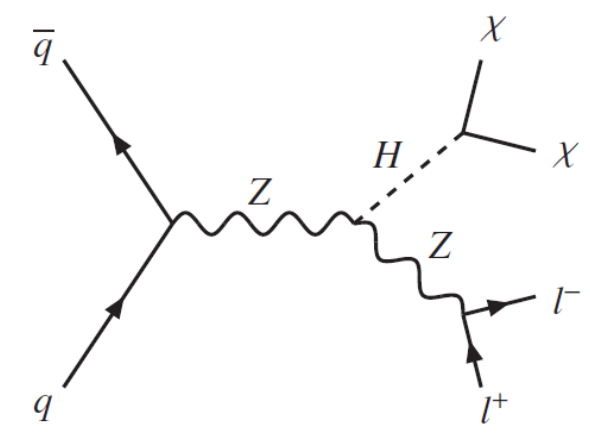

- $Z+$ +jets

ATLAS-CONF-2013-011

After just Z selection:

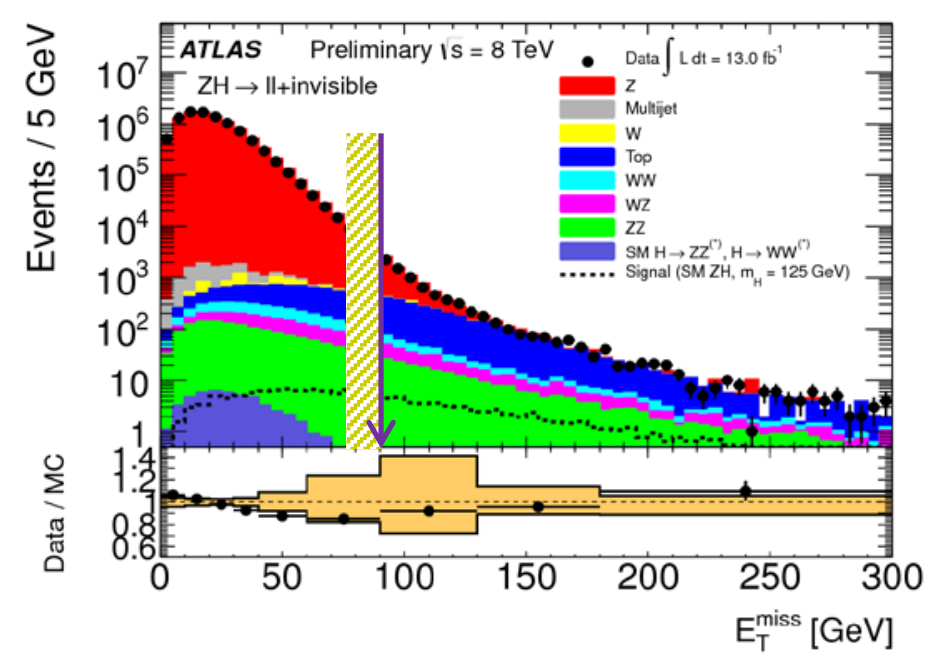

After final selection (8 TeV shown):

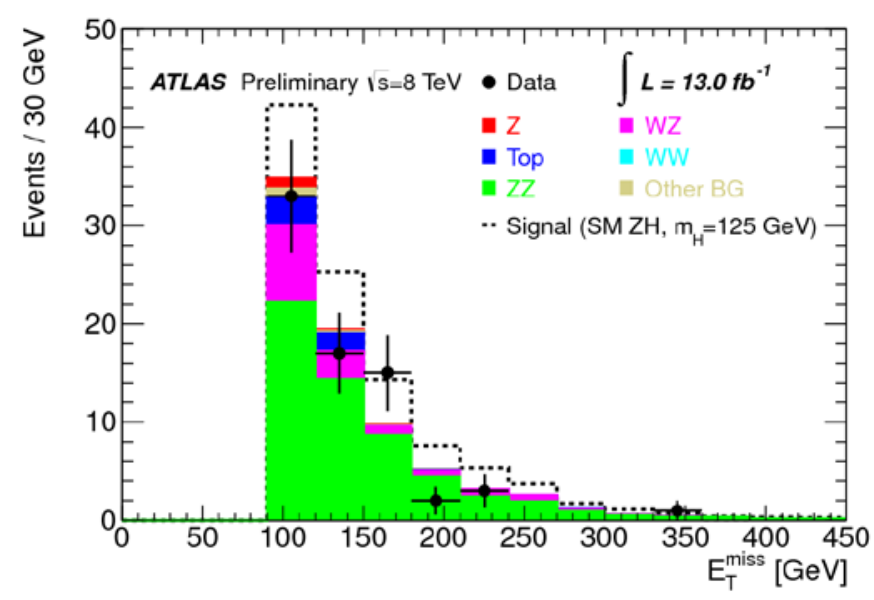

- azimuthal separation

- MET balancing

- veto on additional jets 

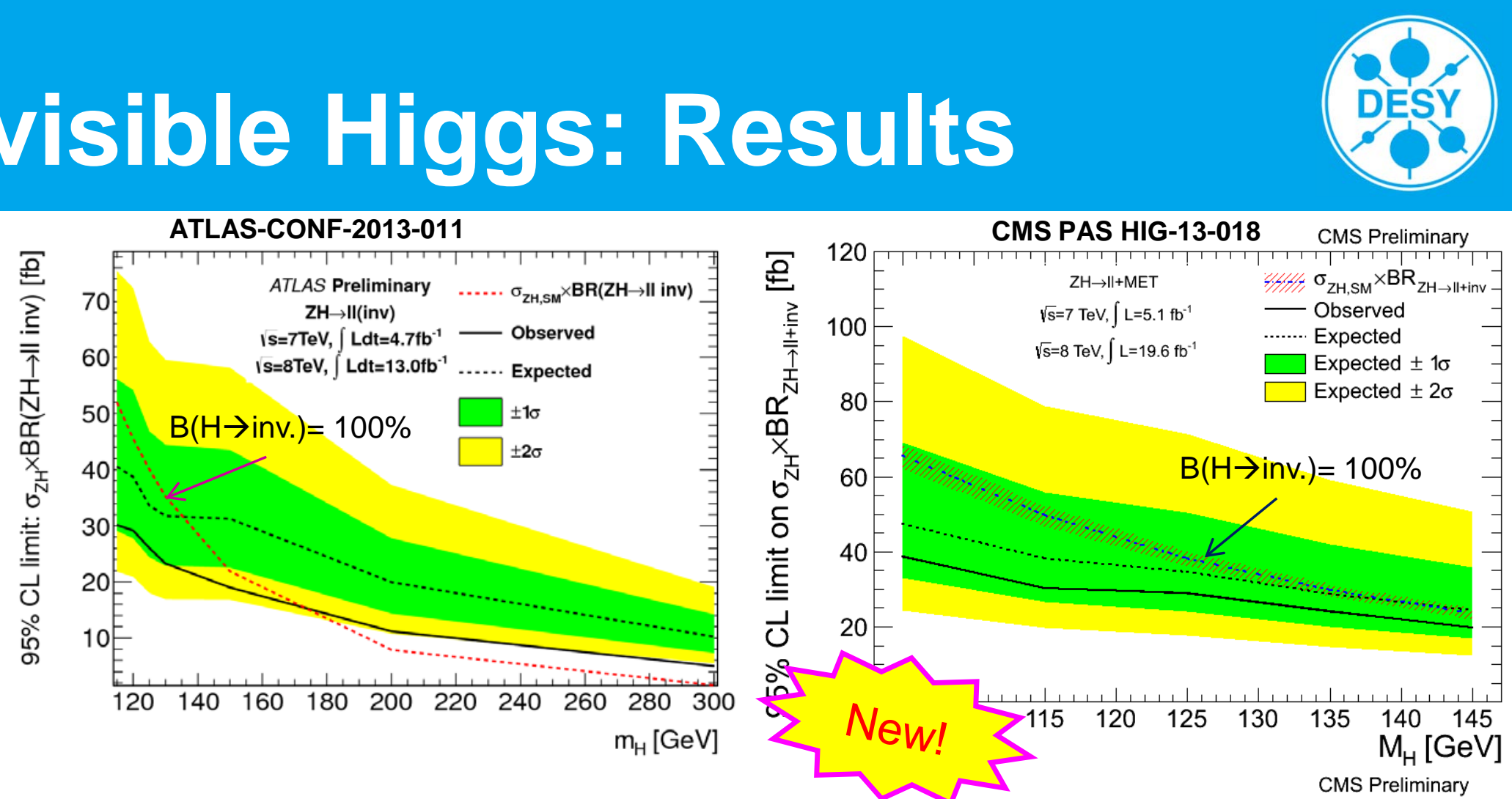

- Invisible BR $<65 \%$ (ATLAS) and $<75 \%$ (CMS) for SM Higgs @ $125 \mathrm{GeV}$ (95\% C.L.)

$\rightarrow$ Leaves plenty of room for invisible decay modes

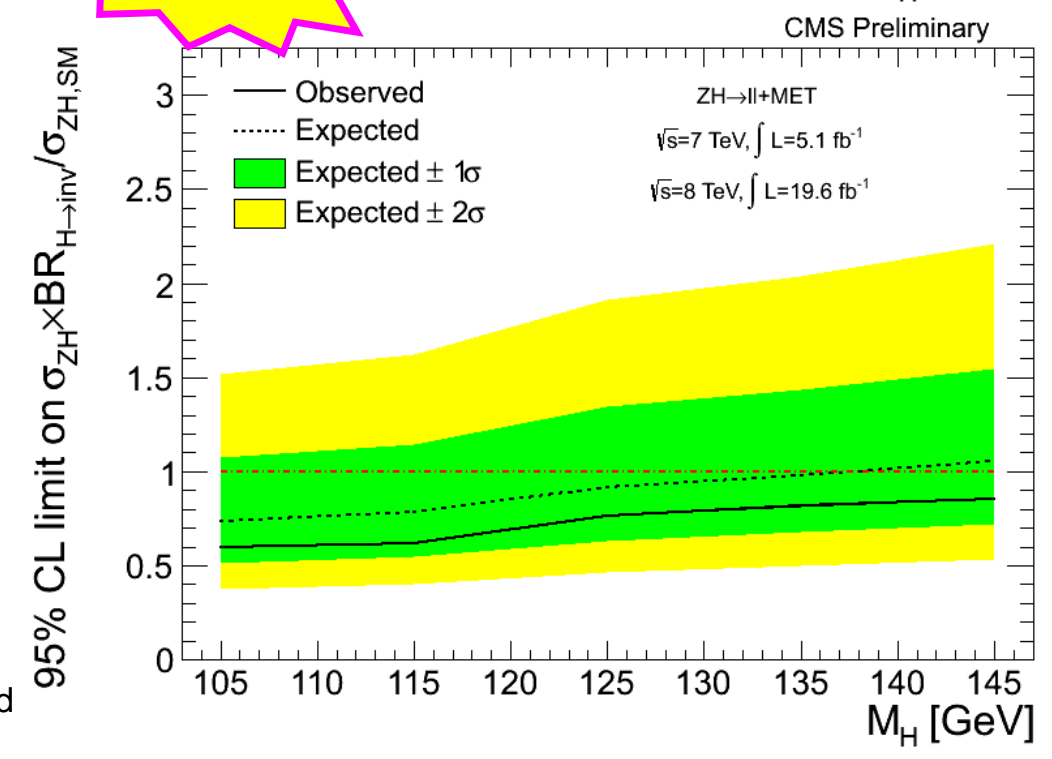


- Invisible Higgs can also be searched in VBF

- cross section higher than in $\mathrm{ZH}$ production

- special VBF+MET triggers

- large efforts to reduce QCD background

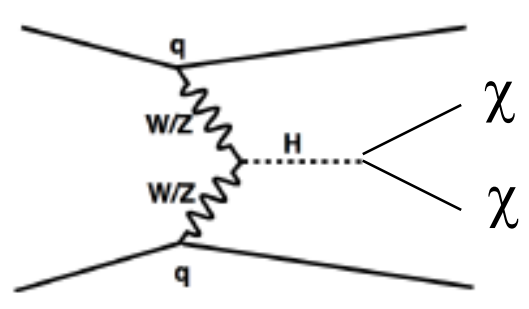

CMS PAS HIG-13-013
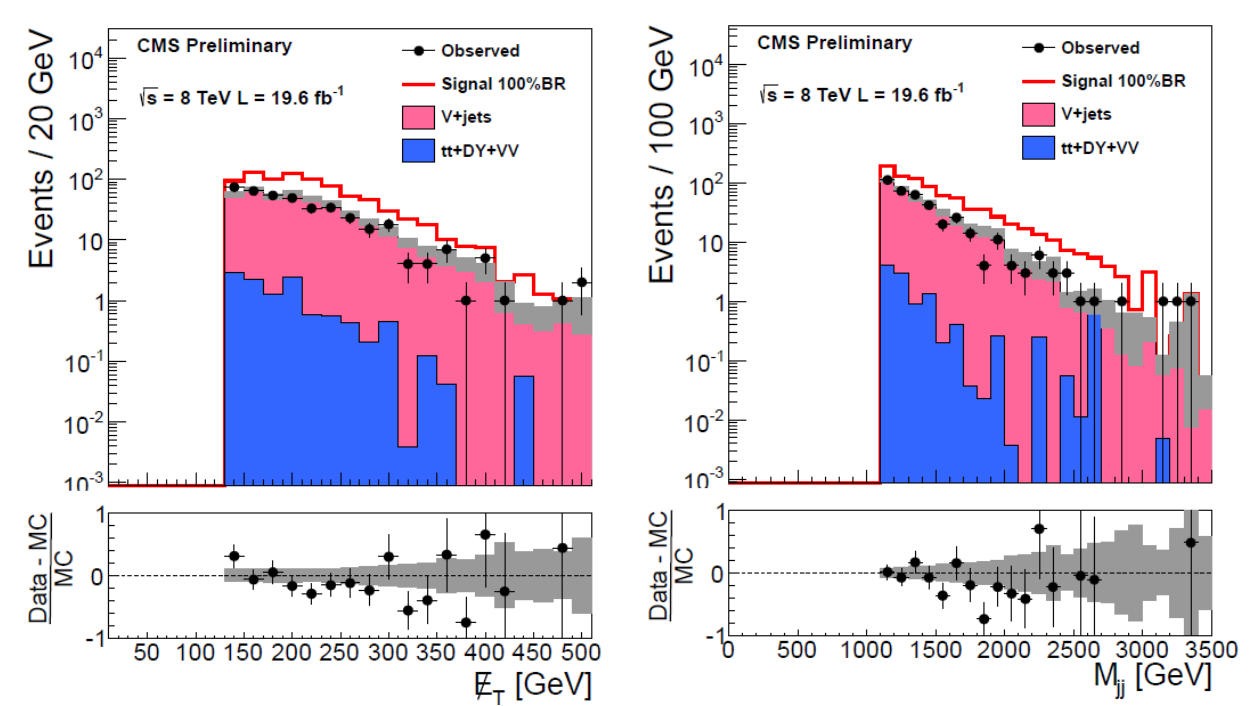

$\rightarrow$ Data in good agreement with SM backgrounds (mainly V+jets)

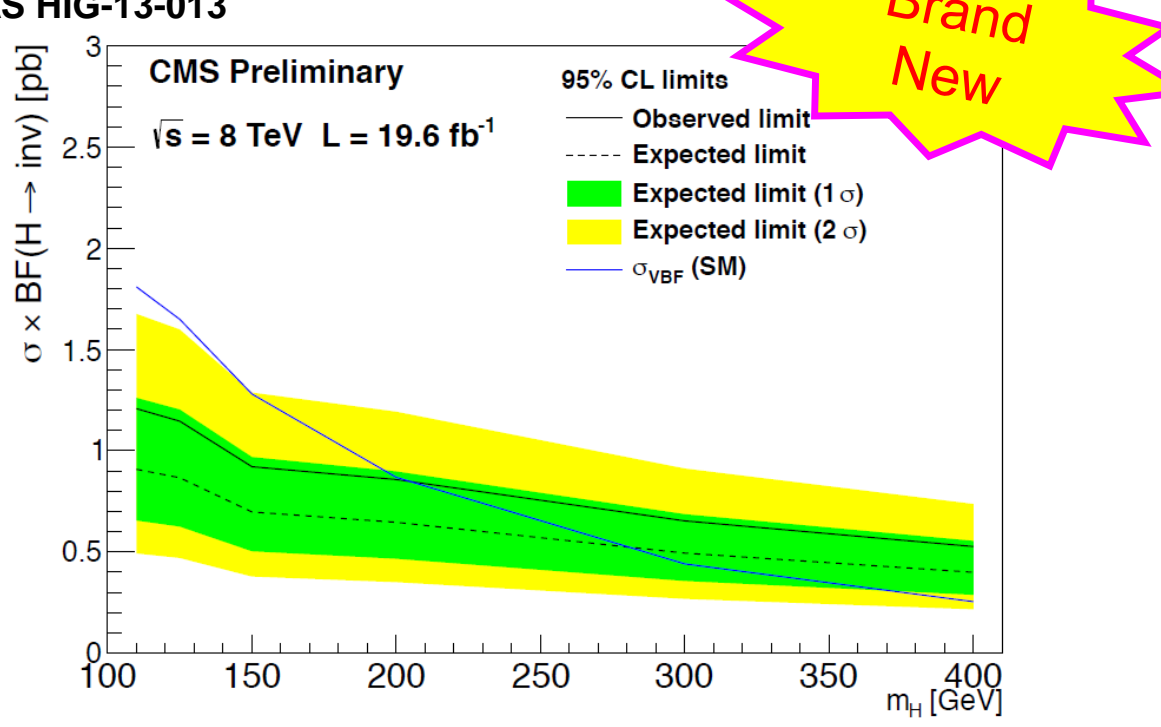

$\rightarrow \quad$ Invisible BR $<69 \%$ for SM Higgs @ $125 \mathrm{GeV}$ (95\% C.L.) 


\section{Fermio-Phobic Higgs}

- If a Higgs boson does not couple to fermions

$\rightarrow$ production via gluon-gluon fusion impossible

$\rightarrow \quad$ standard production channel is vector boson fusion (VBF) or vector-boson associated production $(\mathrm{VH})$

$\rightarrow \quad$ BRs for di-boson modes enhanced

- Analysis largely similar to SM analysis

$\rightarrow$ Fermio-phobic Higgs excluded within $m_{H}=100-147 \mathrm{GeV}$

\section{ATLAS CONF-2012-013}
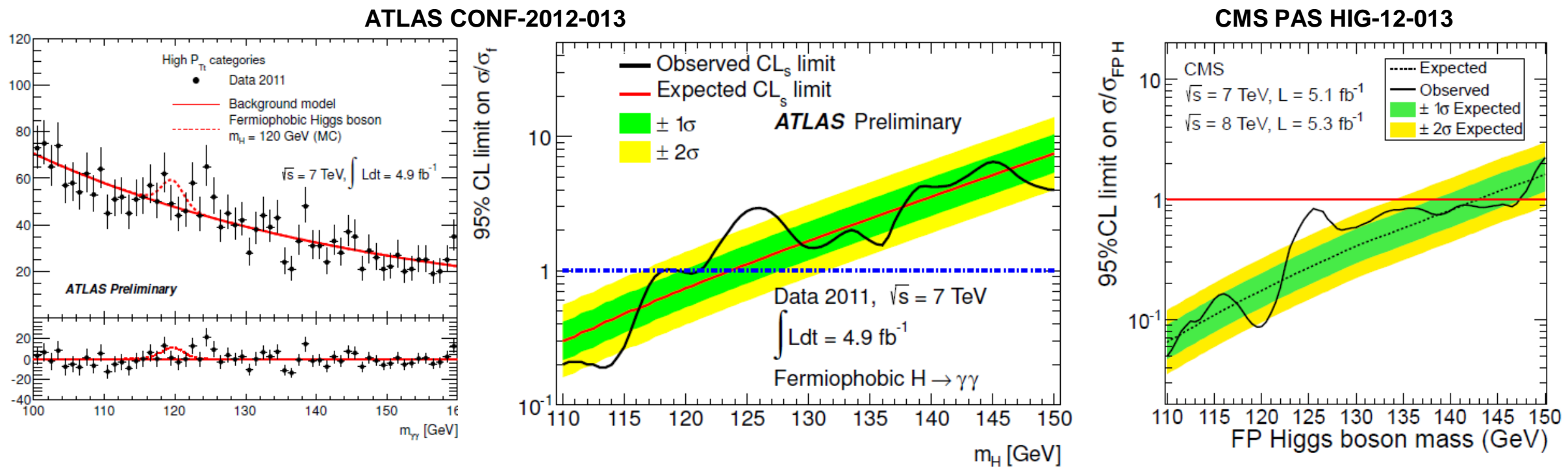
- Observation of SM-like features of the $\mathrm{H}(125)$ state does not imply that the Higgs sector must have SM structure

- best way of clarification: direct search for additional Higgs signatures

- A broad attack is launched to clarify whether the Higgs sector reaches beyond the Standard Model.

- MSSM: at low $m_{\mathrm{A}}(<140 \mathrm{GeV})$ LHC \& LEP limits close. Large $\mathrm{m}_{\mathrm{A}}$ and $\tan \beta$ still possible. Improved constraints for $\mathrm{H}^{ \pm}$

- 2 HDM: constraints in ( $\cos \alpha, \tan \beta)$ space. First searches in cascade decays

- NMSSM: wide open range of possibilities.

- only few channels/signatures addressed so far.

- already relatively stringent limits for light CP-odd Higgs bosons

- additional analyses are underway.

- also Dark SUSY interpretation possible

- Invisible Higgs: first limits obtained in associated production and VBF. Still large BR(H $\rightarrow$ inv $)$ possible.

- Fermio-phobic Higgs excluded within $100-147 \mathrm{GeV}$ 


\section{Outlook}

- Non-SM Higgs searches have just scratched the surface

- many LHC BSM analyses still need to be updated with full $8 \mathrm{TeV}$ statistics

- many additional new analyses underway

- $\quad 13 \mathrm{TeV}$ running will further extend the reach towards higher masses

- A rich research program for the future 